\title{
Computing Topological Persistence for Simplicial Maps
}

\author{
Tamal K. Dey* $\quad$ Fengtao Fan ${ }^{\dagger} \quad$ Yusu Wang ${ }^{\ddagger}$
}

\begin{abstract}
Algorithms for persistent homology and zigzag persistent homology are well-studied for persistence modules where homomorphisms are induced by inclusion maps. In this paper, we propose a practical algorithm for computing persistence under $\mathbb{Z}_{2}$ coefficients for a sequence of general simplicial maps and show how these maps arise naturally in some applications of topological data analysis.

First, we observe that it is not hard to simulate simplicial maps by inclusion maps but not necessarily in a monotone direction. This, combined with the known algorithms for zigzag persistence, provides an algorithm for computing the persistence induced by simplicial maps.

Our main result is that the above simple minded approach can be improved for a sequence of simplicial maps given in a monotone direction. A simplicial map can be decomposed into a set of elementary inclusions and vertex collapses-two atomic operations that can be supported efficiently with the notion of simplex annotations for computing persistent homology. A consistent annotation through these atomic operations implies the maintenance of a consistent cohomology basis, hence a homology basis by duality. While the idea of maintaining a cohomology basis through an inclusion is not new, maintaining them through a vertex collapse is new, which constitutes an important atomic operation for simulating simplicial maps. Annotations support the vertex collapse in addition to the usual inclusion quite naturally.

Finally, we exhibit an application of this new tool in which we approximate the persistence diagram of a filtration of Rips complexes where vertex collapses are used to tame the blow-up in size.
\end{abstract}

\section{Introduction}

Several applications in topological data analysis encounter the following problem: when a simplicial complex $K_{1}$ is modified to another complex $K_{2}$, how do the topological features change. If the modification pertains only to inclusions, that is, $K_{1} \subseteq K_{2}$, one can quantify the changes by the persistent homology group. This idea of topological persistence, originally introduced in [19], has been explored extensively both algebraically and algorithmically in the past decade, see e.g. [3, 5, 7, 9, 11, 18, 23, 25]. When the modification is more general than the inclusions, modeled by considering the map $K_{1} \rightarrow K_{2}$ to be a simplicial map instead of an inclusion map, the status is not the same. In this paper, we present an efficient algorithm for computing topological persistence for simplicial maps and show its application to a problem in topological data analysis.

Traditional persistent homology is defined for a monotone sequence of homomorphisms, where all the maps $K_{i} \rightarrow K_{i+1}$ are along the same direction. In [8], Carlsson and de Silva introduced the zigzag persistence defined for a zigzagging sequence of homomorphisms containing maps both of the form $K_{i} \rightarrow K_{i+1}$

\footnotetext{
${ }^{*}$ Department of Computer Science and Engineering, The Ohio State University, Columbus, OH 43210, USA. Email: tamaldey@cse.ohio-state.edu

${ }^{\dagger}$ Department of Computer Science and Engineering, The Ohio State University, Columbus, OH 43210, USA. Email: fanflcse.ohio-state.edu

${ }^{\ddagger}$ Department of Computer Science and Engineering, The Ohio State University, Columbus, OH 43210, USA. Email: yusuccse.ohio-state.edu
} 
and $K_{i} \leftarrow K_{i+1}$. They also presented a generic prototype algorithm for computing zigzag persistence induced by general homomorphisms. It requires an explicit representation of the homomorphisms between the homology groups of two consecutive complexes in a sequence. In particular, if the input is given in terms of maps between input spaces such as a continuous map $f: K_{i} \rightarrow K_{i+1}$, a representation of the induced homomorphism $f_{*}: H_{*}\left(K_{i}\right) \rightarrow H_{*}\left(K_{i+1}\right)$ between the homology groups needs to be computed. Often this step is costly and, in general, leads to $O\left(n^{4}\right)$ algorithm where each input complex has $O(n)$ simplices. In contrast, when the map $f$ is an inclusion, the persistence algorithm computes the persistent homology in $O\left(n^{3}\right)$ time where $n$ is the total number of simplices inserted.

Using classical algebraic topological concepts such as mapping cylinders, it is not hard to simulate a simplicial map $f: K_{i} \rightarrow K_{i+1}$ by zigzag inclusions through an intermediate complex $\hat{K}$ built from $K_{i}$. However, the complex $\hat{K}$, if constructed naïvely, may have a huge size. As detailed in section 2.3, one can improve upon this naïve construction which converts the input zigzag filtration connected by simplicial maps to another zigzag filtration connected only by inclusion maps. One can then take advantage of the efficient algorithms to compute the persistence diagram for an inclusion-induced zigzag filtration [9, 23].

Our main result detailed in Sections 3 and 4 is that when the input filtration is connected by a monotone (i.e, non-zigzag) sequence of simplicial maps, we can improve further upon the above construction by taking advantage of annotations introduced recently in [4]. One of the main advantages of this approach is that it avoids the detour through $\hat{K}$, and thus requires far fewer operations to move from $K_{i}$ to $K_{i+1}$; see Figure 2 Furthermore, the main auxiliary structure this new direct approach avails is a set of binary bits (elements of $\mathbb{Z}_{2}$ ) attached to simplices which together can be viewed as a single binary matrix. This is in contrast to the simple-minded coning approach which uses the zigzag persistence algorithm [9] that requires multiple such matrices.

One key aspect of our annotation based approach is that it lets us simulate the simplicial maps by a sequence of inclusions and vertex collapses in monotone direction without zigzag. An annotation is linked with a cohomology basis which by duality corresponds to a homology basis. Thus, annotations over inclusions and vertex collapses allow us to maintain a consistent homology basis indirectly under simplicial maps and infer the persistent homology. Our handling of inclusions can be seen as an alternative formulation of the algorithm for computing persistent cohomology proposed in [16]. However, the handling of vertex collapses (which are neither inclusions nor deletions) in the context of persistence is new, and has not been addressed previously.

Finally, in Section 6, we show an application where the need for computing persistence under simplicial maps arises naturally. Our algorithm from Section 4 can be used for this application directly. It is known that the persistence diagram [12] of Vietoris-Rips (Rips in short) filtrations provides avenues for topological analysis of data [1, 14, 20]. However, the inclusive nature of Rips complexes makes its size too huge to be taken advantage of in practice. One can consider sparsified versions of Rips complexes [24] or graph induced complexes [13] by subsampling input points which can be achieved by vertex collapses. Our algorithm supports vertex collapses and thus naturally yields to maps arising out of such subsampling.

Throughout the paper, simplicial homology and cohomology groups are defined with coefficients in $\mathbb{Z}_{2}$.

\section{Preliminaries and simplicial maps}

Definition 2.1 Given a finite set $V$, a simplicial complex $K=K(V)$ is defined as a collection of subsets $\{\sigma \subseteq V\}$ so that $\sigma \in K$ implies that any subset $\sigma^{\prime} \subseteq \sigma$ is in $K$. The vertex set $V(K)$ of $K$ is $V$. The elements of $K$ are called its simplices. An element $\sigma \in K$ is a p-simplex if its cardinality is $p+1$. A simplex $\sigma^{\prime}$ is a face of $\sigma$ and $\sigma$ is a coface of $\sigma^{\prime}$ if $\sigma^{\prime} \subseteq \sigma$.

Definition 2.2 Let $X$ be a subset of a simplicial complex $K$. The set $\operatorname{St} X:=\left\{\sigma^{\prime} \in K \mid \exists \sigma \in X\right.$ and $\left.\sigma \subseteq \sigma^{\prime}\right\}$ 
is called the star of $X$. The closure of $X$, denoted $\bar{X}$, is the simplicial complex formed by simplices in $X$ and all of their faces. The link of $X$ is $\mathrm{L} k X:=\overline{\mathrm{St} X} \backslash \operatorname{St} \bar{X}$.

The star of $X$ consists of the simplices in $K$ that are cofaces of simplices in $X$. The link of $X$ consists of the faces of the simplices in its star which contain no vertex of $X$.

\section{1 (Co)homology groups}

We briefly introduce the notion of homology and cohomology groups here which we use extensively; see e.g. Hatcher [22] for details. Both groups are defined under $\mathbb{Z}_{2}$ coefficients. A $p$-chain $c_{p}$ in a simplicial complex $K$ is a formal sum of $p$-simplices, that is, $c_{p}=\Sigma \alpha_{i} \sigma_{i}, \alpha_{i} \in\{0,1\}$ and $\sigma_{i} \in K$. The chains under $\mathbb{Z}_{2}$-additions form an abelian group called the $p$-chain group of $K$ and is denoted $C_{p}(K)$. The boundary of a $p$-simplex $\sigma$, denoted $\partial_{p} \sigma$, is defined to be the formal sum of its boundary $(p-1)$-simplices. We obtain the boundary homomorphism $\partial_{p}: C_{p} \rightarrow C_{p-1}$ given by $\partial_{p}\left(\Sigma \alpha_{i} \sigma_{i}\right)=\Sigma \alpha_{i}\left(\partial_{p} \sigma_{i}\right)$. The kernel of $\partial_{p}$ is the cycle group $Z_{p} \subseteq C_{p}$. The image of $\partial_{p}$ is the boundary group $B_{p-1} \subseteq C_{p-1}$. It can be easily verified that $\partial_{p} \circ \partial_{p-1}=0$ which makes the quotient group $H_{p}(K)=Z_{p}(K) / B_{p}(K)$, known as the $p$ th homology group, well defined.

Cohomology groups are defined by cochains, cocycles, and coboundaries that are, in a sense, functional duals to the chains, cycles, and boundaries respectively. A $p$-cochain is a homomorphism $c^{p}: C_{p}(K) \rightarrow \mathbb{Z}_{2}$ and thus can be completely specified by its value on each $p$-simplex. The $p$-cochain group $C^{p}(K)$ is the group of all cochains under $\mathbb{Z}_{2}$-additions. The coboundary operator $\delta_{p}: C^{p} \rightarrow C^{p+1}$ sends $p$-cochains to $(p+1)$-cochains by evaluating $\delta_{p} c^{p}$ on each chain $d_{p+1} \in C_{p+1}$ as $c^{p}\left(\partial_{p+1} d_{p+1}\right)$. The kernel of $\delta_{p}$ is the cocycle group $Z^{p}(K)$ and its image is the coboundary group $B^{p+1}(K)$. Since $\delta_{p} \circ \delta_{p+1}=0$, we have the quotient group $Z^{p}(K) / B^{p}(K)$ well defined which is called the cohomology group $H^{p}(K)$.

\subsection{Simplicial maps}

Definition 2.3 A map $f: K \rightarrow K^{\prime}$ is simplicial if for every simplex $\sigma=\left\{v_{0}, v_{1}, \ldots, v_{k}\right\}$ in $K, f(\sigma)=$ $\left\{f\left(v_{0}\right), f\left(v_{1}\right), \ldots, f\left(v_{k}\right)\right\}$ is a simplex in $K^{\prime}$. The restriction $f_{V}$ of $f$ to $V(K)$ is a vertex map.

A simplicial map $f: K_{1} \rightarrow K_{2}$ induces a homomorphism $H_{p}\left(K_{1}\right) \stackrel{f_{*}}{\rightarrow} H_{p}\left(K_{2}\right)$ for the homology groups in the forward direction while a homomorphism $H^{p}\left(K_{1}\right) \stackrel{f^{*}}{\leftarrow} H^{p}\left(K_{2}\right)$ for the cohomology groups in the backward direction. The latter sends a cohomology class $[c]$ in $H^{p}\left(K_{2}\right)$ to the cohomology class $\left[c^{\prime}\right]$ in $H^{p}\left(K_{1}\right)$ where $c^{\prime}\left(c_{p}\right)=c\left(f\left(c_{p}\right)\right)$ for each $c_{p} \in C_{p}\left(K_{1}\right)$.

Definition 2.4 A simplicial map $f: K \rightarrow K^{\prime}$ is called elementary if it is of one of the following two types:

- $f$ is injective, and $K^{\prime}$ has at most one more simplex than $K$. In this case, $f$ is called an elementary inclusion.

- $f$ is not injective but is surjective, and the vertex map $f_{V}$ is injective everywhere except on a pair $\{u, v\} \subseteq V(K)$. In this case, $f$ is called an elementary collapse. An elementary collapse maps a pair of vertices into a single vertex, and is injective on every other vertex.

We observe that any simplicial map is a composition of elementary simplicial maps (see Appendix A).

Proposition 2.5 If $f: K \rightarrow K^{\prime}$ is a simplicial map, then there are elementary simplicial maps $f_{i}$

$$
K \stackrel{f_{1}}{\rightarrow} K_{1} \stackrel{f_{2}}{\rightarrow} K_{2} \cdots \stackrel{f_{n}}{\rightarrow} K_{n}=K^{\prime} \text { so that } f=f_{n} \circ f_{n-1} \circ \cdots \circ f_{1} .
$$


In view of Proposition 2.5, it is sufficient to show how one can design the persistence algorithm for an elementary simplicial map. At this point, we make a change in the definition 2.4 of elementary simplicial maps that eases further discussions. We let $f_{\mathrm{V}}$ to be identity (which is an injective map) everywhere except possibly on a pair of vertices $\{u, v\} \subseteq V(K)$ for which $f_{\mathrm{V}}$ maps to a single vertex, say $u$ in $K^{\prime}$. This change can be implemented by renaming the vertices in $K^{\prime}$ that are mapped onto injectively. Since the standard persistence algorithm handles inclusions, we focus mainly on handling the elementary collapses.

\subsection{Simulation with coning}

First, we propose a simulation of simplicial maps with a coning strategy that only requires additions of simplices. We focus on elementary collapses. Let $f: K \rightarrow K^{\prime}$ be an elementary collapse. Assume that

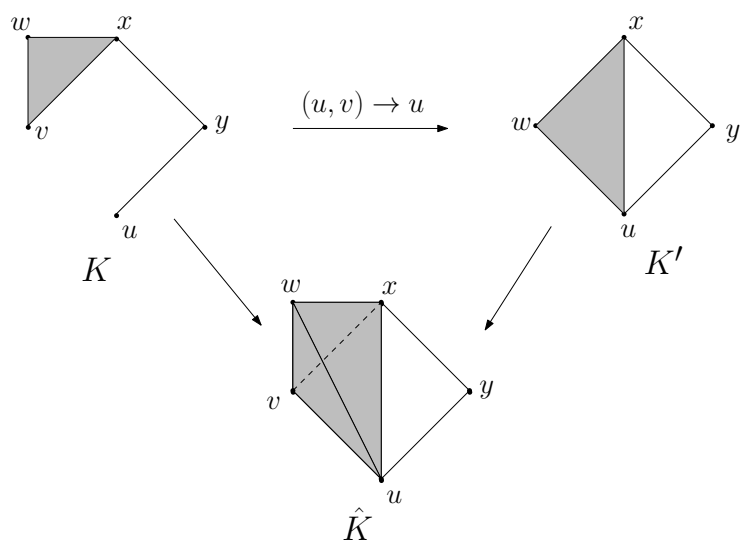

Figure 1: Elementary collapse $(u, v) \rightarrow u$ : the cone $u * \overline{\operatorname{St} v}$ adds edges $\{u, w\},\{u, v\},\{u, x\}$, triangles $\{u, w, x\},\{u, v, x\},\{u, v, w\}$, and the tetrahedron $\{u, v, w, x\}$.

the induced vertex map collapses vertices $u, v \in K$ to $u \in K^{\prime}$, and is identity on other vertices. For a subcomplex $X \subseteq K$, define the cone $u * X$ to be the complex $\{\overline{\sigma \cup\{u\}} \mid \sigma \in X\}$. Consider the augmented complex

$$
\hat{K}:=K \cup(u * \overline{\operatorname{St} v}) .
$$

In other words, for every simplex $\left\{u_{0}, \ldots, u_{d}\right\} \in \overline{\operatorname{St} v}$ of $K$, we add the simplex $\left\{u_{0}, \ldots, u_{d}\right\} \cup\{u\}$ to $\hat{K}$ if it is not already in. See Figure 11. Notice that $K^{\prime}$ is a subcomplex of $\hat{K}$ in this example which we observe is true in general.

Claim 2.6 $K^{\prime} \subseteq \hat{K}$.

Proof: For a simplex $\sigma \in K^{\prime}$ that does not contain $u, f$ is identity on its unique pre-image; that is, $\sigma \in K \subseteq$ $\hat{K}$. Now consider a $d$-simplex $\sigma=\left\{u, u_{1}, \ldots, u_{d}\right\} \in K^{\prime}$. Since $f$ is surjective, there exists at least one pre-image of $\sigma$ in $K$ of the form $\sigma^{\prime}=\left\{u_{0}, u_{1}, \ldots, u_{d}\right\}$, where $u_{0}$ is either $u$ or $v$. If it is $u_{0}=u$, we have $f\left(\sigma^{\prime}\right)=\sigma^{\prime}=\sigma$ and thus $\sigma \in K \subseteq \hat{K}$. So, assume that $u_{0}=v$. This means that the simplex $\left\{u_{1}, \ldots, u_{d}\right\}$ is in Lkv (and thus in $\overline{\mathrm{St} v}$ ), implying that $\sigma=\left\{u, u_{1}, \ldots, u_{d}\right\} \in \hat{K}$.

Now consider the canonical inclusions $i: K \hookrightarrow \hat{K}$ and $i^{\prime}: K^{\prime} \hookrightarrow \hat{K}$. These inclusions constitute the diagram on the righthand side which does not necessarily commute. Nevertheless, it commutes at the homology level which is precisely stated below.

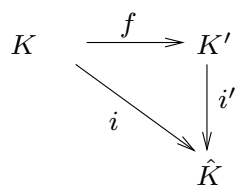




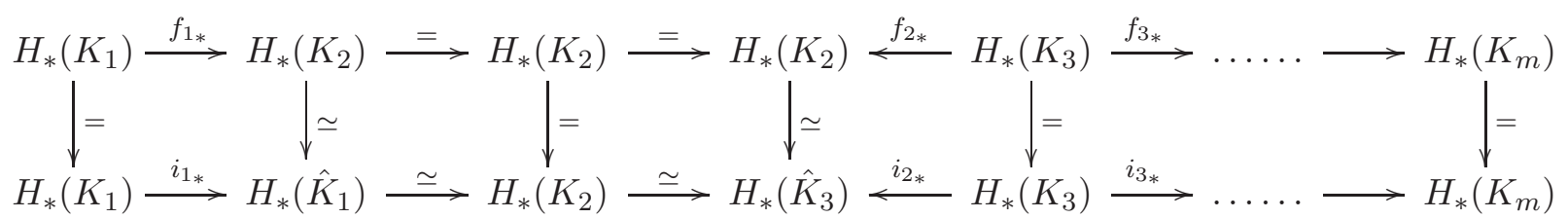

Proposition $2.7 f_{*}: H_{*}(K) \rightarrow H_{*}\left(K^{\prime}\right)$ is equal to $\left(i_{*}^{\prime}\right)^{-1} \circ i_{*}$ where $i_{*}^{\prime}$ is an isomorphism and $H_{*}(K) \stackrel{i_{*}}{\rightarrow}$ $H_{*}(\hat{K}) \stackrel{i_{*}^{\prime}}{\leftarrow} H_{*}\left(K^{\prime}\right)$.

Proof: We use the notion of contiguous maps which induces equal maps at the homology level. Two maps $f_{1}: K_{1} \rightarrow K_{2}, f_{2}: K_{1} \rightarrow K_{2}$ are contiguous if for every simplex $\sigma \in K_{1}, f_{1}(\sigma) \cup f_{2}(\sigma)$ is a simplex in $K_{2}$. We observe that the simplicial maps $i^{\prime} \circ f$ and $i$ are contiguous and $i^{\prime}$ induces an isomorphism at the homology level, that is, $i_{*}^{\prime}: H_{*}(K) \rightarrow H_{*}(\hat{K})$ is an isomorphism.

Since $i$ is contiguous to $i^{\prime} \circ f$ (Proposition A.1 in appendix), we have $i_{*}=\left(i^{\prime} \circ f\right)_{*}=i_{*}^{\prime} \circ f_{*}$. Since $i_{*}^{\prime}$ is an isomorphism (Proposition A.2 in appendix), $\left(i_{*}^{\prime}\right)^{-1}$ exists and is an isomorphism. It then follows that $f_{*}=\left(i_{*}^{\prime}\right)^{-1} \circ i_{*}$.

Proposition 2.7 allows us to simulate the persistence of a sequence of simplicial maps with only inclusioninduced homomorphisms. Consider the following sequence of simplicial complexes connected with a zigzag sequence of simplicial maps (the arrows could be oriented differently in general):

$$
K_{1} \stackrel{f_{1}}{\rightarrow} K_{2} \stackrel{f_{2}}{\leftarrow} K_{3} \stackrel{f_{3}}{\rightarrow} \cdots \rightarrow K_{n}
$$

which generates the module at the homology level by induced homomorphisms $f_{i *}$

$$
\mathcal{F}: H_{*}\left(K_{1}\right) \stackrel{f_{1_{*}}}{\rightarrow} H_{*}\left(K_{2}\right) \stackrel{f_{2_{*}}}{\leftarrow} H_{*}\left(K_{3}\right) \stackrel{f_{3 *}}{\rightarrow} \cdots \rightarrow H_{*}\left(K_{n}\right)
$$

When the maps $f_{i}$ are all inclusions, it is known that the zigzag persistence induced by them can be computed in matrix multiplication time by a recent algorithm of [23]. This algorithm does not extend to simplicial maps as per se though we know that a persistence module induced by simplicial maps admits a decomposition [25] and hence a persistence diagram [12]. With our observation that every simplicial map can be simulated with inclusion maps, we can take advantage of the algorithm of [23] for computing zigzag persistence for simplicial maps. In view of Proposition 2.7, consider the following sequence connected only with inclusions:

$$
K_{1} \hookrightarrow \hat{K}_{1} \hookleftarrow K_{2} \hookrightarrow \hat{K}_{3} \hookleftarrow K_{3} \hookrightarrow \cdots \hookleftarrow K_{n}
$$

At the homology level we have $H_{*}\left(\hat{K}_{i}\right) \simeq H_{*}\left(K_{i+1}\right)$ induced by the inclusion $\hat{K}_{i} \hookleftarrow K_{i+1}$ and also $H_{*}\left(K_{i}\right) \simeq H_{*}\left(\hat{K}_{i+1}\right)$ induced by the inclusion $K_{i} \hookrightarrow \hat{K}_{i+1}$. Thus, we have the following persistence module:

$$
\begin{aligned}
\mathcal{M}: H_{*}\left(K_{1}\right) & \stackrel{i_{*}}{\rightarrow} H_{*}\left(\hat{K}_{1}\right) \\
& \simeq H_{*}\left(K_{2}\right) \\
& \simeq H_{*}\left(\hat{K}_{3}\right) \stackrel{i_{*}}{\leftarrow} H_{*}\left(K_{3}\right) \stackrel{i_{*}}{\rightarrow} \cdots \stackrel{i_{*}}{\leftarrow} H_{*}\left(K_{n}\right)
\end{aligned}
$$

Theorem 2.8 The persistence diagram of $\mathcal{F}$ can be derived from the that of the module $\mathcal{M}$.

Proof: Consider the diagram between vector spaces as shown above. All isomorphisms are induced by inclusions, hence every square being supported only by isomorphisms commutes. The other squares supported by $f_{i_{*}}$ also commute because of Proposition 2.7. Hence every square in this diagram commutes, and the claim follows [8, 18]. 


\section{Annotations}

When we are given a non-zigzag sequence of simplicial maps $K_{1} \stackrel{f_{1}}{\rightarrow} K_{2} \stackrel{f_{2}}{\rightarrow} K_{3} \cdots \stackrel{f_{n}}{\rightarrow} K_{n}$ we can improve upon the coning approach by reducing simplex insertions as illustrated in Figure 2 Consider the map $f_{i j}$ : $K_{i} \rightarrow K_{j}$ where $f_{i j}=f_{j-1} \circ \cdots \circ f_{i+1} \circ f_{i}$. To compute the persistent homology, the persistence algorithm essentially maintains a consistent basis by computing the image $f_{i j_{*}}\left(B_{i}\right)$ of a basis $B_{i}$ of $H_{*}\left(K_{i}\right)$. As one moves through a map in the filtration, the homology basis elements get created (birth) or can be interpreted to be destroyed (death). The notion of this birth and death of the homology basis elements can be formulated precisely with algebra [25] and can be summarized with persistence diagrams [12]. Here, instead of a consistent homology basis, we maintain a consistent cohomology basis, that is, if $B^{i}$ is a cohomology basis of $H^{*}\left(K_{i}\right)$ maintained by the algorithm, we compute the preimage $f_{i j}^{*-1}\left(B^{i}\right)$ where $H^{*}\left(K_{i}\right) \stackrel{f_{i j}^{*}}{\leftarrow} H^{*}\left(K_{j}\right)$ is the homomorphism induced in the cohomology groups by $f_{i j}$. By duality, this implicitly maintains a consistent homology basis and thus captures all information about persistent homology as well [16].

Our main tool to maintain a consistent cohomology basis is the notion of annotation [4] which are binary vectors assigned to simplices. We maintain the annotations as we go forward through the given sequence, and thus maintain a cohomology basis in the reverse direction whose birth and death coincide with the death and birth respectively of a consistent homology basis.

Definition 3.1 Given a simplicial complex $K$, Let $K(p)$ denote the set of p-simplices in $K$. An annotation for $K(p)$ is an assignment $\mathrm{a}: K(p) \rightarrow \mathbb{Z}_{2}^{g}$ of a binary vector $\mathrm{a}_{\sigma}=\mathrm{a}(\sigma)$ of same length $\mathrm{g}$ for each $p$-simplex $\sigma \in K$. Entries of $\mathrm{a}_{\sigma}$ are called its elements. We also have an induced annotation for any $p$-chain $c_{p}$ given by $\mathrm{a}_{c_{p}}=\Sigma_{\sigma \in c_{p}} \mathrm{a}_{\sigma}$.

Definition 3.2 An annotation a : $K(p) \rightarrow \mathbb{Z}_{2}^{g}$ is valid if conditions 1 and 2 are satisfied:

1. $g=\operatorname{rank} H_{p}(K)$, and

2. two p-cycles $z_{1}$ and $z_{2}$ have $\mathrm{a}_{z_{1}}=\mathrm{a}_{z_{2}}$ iff their homology classes are identical, i.e. $\left[z_{1}\right]=\left[z_{2}\right]$.

Proposition 3.3 Statements 1 and 2 are equivalent:

1. An annotation a $: K(p) \rightarrow \mathbb{Z}_{2}^{g}$ is valid

2. The cochains $\left\{\phi_{i}\right\}_{i=1, \cdots, g}$ given by $\phi_{i}(\sigma)=\mathrm{a}_{\sigma}[i]$ for all $\sigma \in K(p)$ are cocycles whose cohomology classes $\left\{\left[\phi_{i}\right]\right\}, i=1, \ldots, g$ constitute a basis of $H^{p}(K)$.

Proof: $1 \rightarrow 2$ : The cochains $\phi_{i}$ are cocycles since for any $(p+1)$-simplex $\tau \in K(p+1)$ one has $[\partial \tau]=[0]$ and hence $\delta_{p} \phi_{i}(\tau)=\phi_{i}(\partial \tau)=\phi_{i}(0)=0$, where $\delta_{p}$ is the co-boundary operator for $p$-dimensional cochains. Let $\left[z_{1}\right],\left[z_{2}\right], \cdots,\left[z_{g}\right]$ be a basis of $H_{p}(K)$. Let $V$ be the vector space generated by $\left[\phi_{i}\right], i=$ $1, \cdots, g$. Define a bilinear form $\alpha: V \times H_{p}(K) \rightarrow \mathbb{Z}_{2}$ by $\alpha\left(\left[\phi_{i}\right],\left[z_{j}\right]\right)=\phi_{i}\left(z_{j}\right)$. The matrix $\left[\phi_{i}\left(z_{j}\right)\right]_{i j}$ has full rank due to the condition 2 in the definition of annotation. This means the vector spaces $V$ and $H_{p}(K)$ have the same rank and hence are isomorphic. It follows that $V \simeq H^{p}(K)$.

$2 \rightarrow 1$ : For this direction, consider a basis $\left[z_{1}\right],\left[z_{2}\right], \cdots,\left[z_{g}\right]$ of $H_{p}(K)$. By universal coefficient theorem we have an isomorphism $H^{p}(K) \simeq \operatorname{Hom}\left(H_{p}(K), \mathbb{Z}_{2}\right)$ which sends a cocycle class $\left[\phi_{i}\right]$ to the homomorphism $\left[z_{j}\right] \mapsto \phi_{i}\left(z_{j}\right)$. This means that the matrix $\left[\phi_{i}\left(z_{j}\right)\right]_{i j}$ has full rank and hence the vectors $\left[\phi_{1}\left(z_{j}\right), \ldots, \phi_{g}\left(z_{j}\right)\right]$ and $\left[\phi_{1}\left(z_{k}\right), \ldots, \phi_{g}\left(z_{k}\right)\right]$ are identical if and only if $\left[z_{i}\right]=\left[z_{k}\right]$. The claim can be extended to any homology class since it can be expressed as a linear combination of the basis elements. 
In light of the above result, an annotation is simply one way to represent a cohomology basis. However, by representing the corresponding basis as an explicit vector associated with each simplex, it localizes the basis to each simplex. As a result, we can update the cohomology basis locally by changing the annotations locally (see Proposition 4.4). This point of view also helps to reveal how we can process elementary collapses, which are neither inclusions nor deletions, by transferring annotations (see Proposition 4.5).

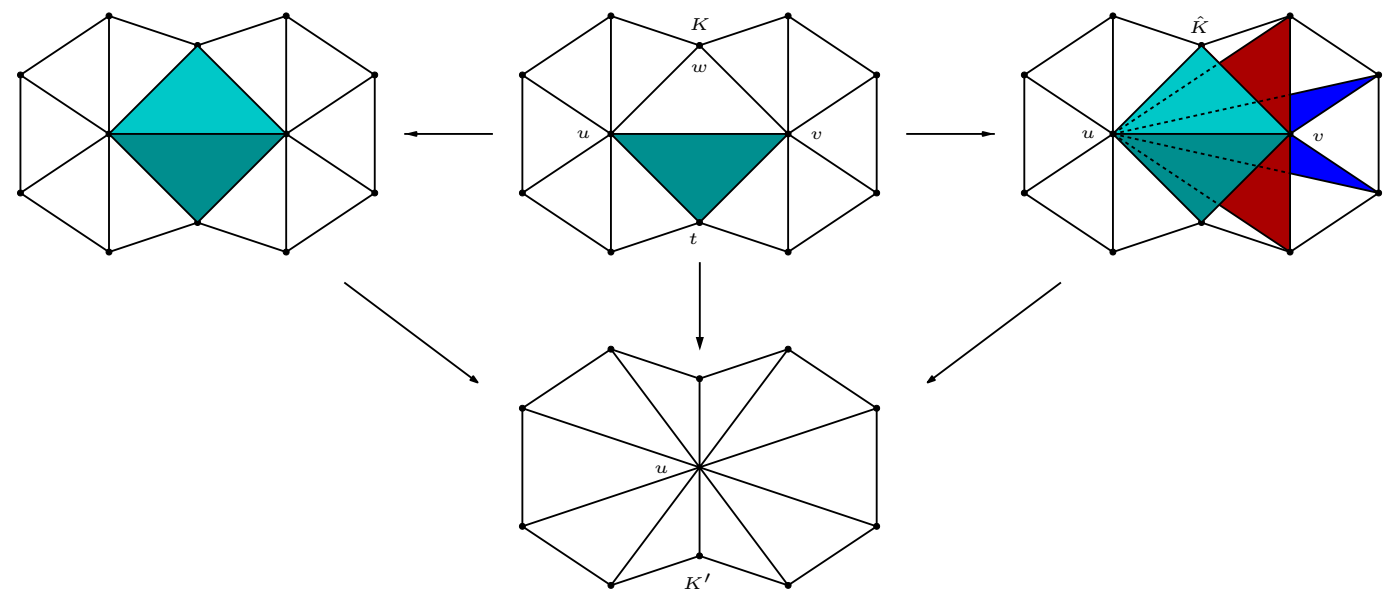

Figure 2: Annotation vs. coning: The pair $(u, v)$ is collapsed to $u$ in $K$ to produce $K^{\prime}$ (middle column). The 2-simplices are the shaded triangles alone. Annotation requires inserting (implicitly) the single triangle as shown on the left whereas coning requires inserting many more simplices as shown by shaded triangles on the right. Specifically, the coning approach requires inserting all simplices in the cone $u * \overline{\mathrm{St}} v$ formed by $u$ and all simplices in the closure of the star of $v$.

\section{Algorithm}

Consider the persistence module $\mathcal{M}$ induced by elementary simplicial maps $f_{i}: K_{i} \rightarrow K_{i+1}$.

$$
\mathcal{M}: H_{*}\left(K_{1}\right) \stackrel{f_{1_{*}}}{\rightarrow} H_{*}\left(K_{2}\right) \stackrel{f_{2_{*}}}{\rightarrow} H_{*}\left(K_{3}\right) \cdots \stackrel{f_{n_{*}}}{\rightarrow} H_{*}\left(K_{n}\right)
$$

Instead of tracking a consistent homology basis for the module $\mathcal{M}$, we track a cohomology basis in the dual module $\mathcal{M}^{*}$ where the homomorphisms are in reverse direction:

$$
\mathcal{M}^{*}: H^{*}\left(K_{1}\right) \stackrel{f_{1}^{*}}{\leftarrow} H^{*}\left(K_{2}\right) \stackrel{f_{2}^{*}}{\leftarrow} H^{*}\left(K_{3}\right) \cdots \stackrel{f_{n}^{*}}{\leftarrow} H^{*}\left(K_{n}\right)
$$

As we move from left to right in the above sequence, the annotations implicitly maintain a cohomology basis whose elements are also time stamped to signify when a basis element is born or dies. We should keep in mind that the birth and death of a cohomology basis element coincides with the death and birth of a homology basis element because the two modules run in opposite directions.

\subsection{Elementary inclusion}

The handling of elementary inclusions using annotations can be viewed as an alternative formulation of the algorithm proposed in [16]; see also [17]. We describe it in terms of the annotation here because it is also used in an elementary collapse, a new atomic operation that we need to address. Consider an elementary inclusion $K_{i} \hookrightarrow K_{i+1}$. Assume that $K_{i}$ has a valid annotation. We describe how we obtain a valid annotation 


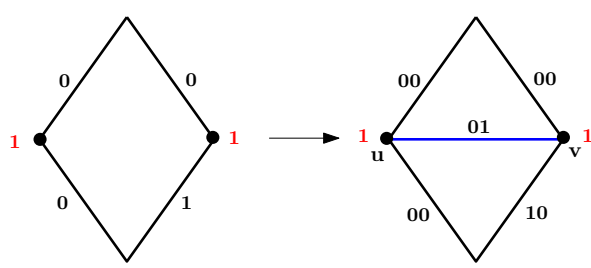

(a) Case(i)

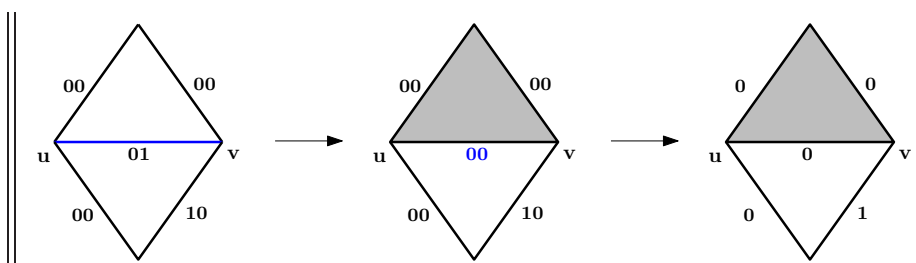

(b) Case(ii)

Figure 3: Case(i) of inclusion: the boundary $\partial u v=u+v$ of the edge $u v$ has annotation $1+1=0$. After its addition, every edge gains an element in its annotation which is 0 for all except the edge $u v$. Case (ii) of inclusion: the boundary of the top triangle has annotation 01. It is added to the annotation of $u v$ which is the only edge having the second element 1 . Consequently the second element is zeroed out for every edge, and is deleted.

for $K_{i+1}$ from that of $K_{i}$ after inserting the $p$-simplex $\sigma=K_{i+1} \backslash K_{i}$. We compute the annotation a $\partial \sigma$ for the boundary $\partial \sigma$ in $K_{i}$ and take actions as follows. A formal justification is provided in Section 5

Case (i): If $\mathrm{a}_{\partial \sigma}$ is a zero vector, the class $[\partial \sigma]$ is trivial in $H_{p-1}\left(K_{i}\right)$. This means $\sigma$ creates a $p$-cycle in $K_{i+1}$ and by duality a $p$-cocycle is killed while going left from $K_{i+1}$ to $K_{i}$. In this case we augment the annotations for all $p$-simplices by one element with a time stamp $i+1$, that is, an annotation $\left[b_{1}, b_{2}, \cdots, b_{g}\right]$ for a $p$-simplex $\tau$ is updated to $\left[b_{1}, b_{2}, \cdots, b_{g}, b_{g+1}\right]$ with the last element time stamped $i+1$ where $b_{g+1}=0$ for $\tau \neq \sigma$ and $b_{g+1}=1$ for $\tau=\sigma$. The element $b_{i}$ of $\mathrm{a}_{\sigma}$ is set to zero for $1 \leq i \leq g$. Other annotations for other simplices remain unchanged. See Figure 3 (a).

Case (ii): If $\mathrm{a}_{\partial \sigma}$ is not a zero vector, the class of the $(p-1)$-cycle $\partial \sigma$ is nontrivial in $H_{p-1}\left(K_{i}\right)$. Therefore, $\sigma$ kills the class of this cycle and a corresponding dual class of cocycles is born in the reverse direction. We simulate it by forcing $\mathrm{a}_{\partial \sigma}$ to be zero which affects other annotations as well. Let $i_{1}, i_{2}, \cdots, i_{k}=u$ be the set of indices in non-decreasing order so that $b_{i_{1}}, b_{i_{2}}, \cdots, b_{i_{k}}=b_{u}$ are all of the nonzero elements in $\mathrm{a}_{\partial \sigma}=\left[b_{1}, b_{2}, \cdots, b_{u}, \cdots, b_{g}\right]$. The cocycle $\phi=\phi_{i_{1}}+\phi_{i_{2}}+\cdots+\left(\phi_{i_{k}}=\phi_{u}\right)$ should become a coboundary after the addition of $\sigma$, which renders

$$
\phi_{u}=\phi_{i_{1}}+\phi_{i_{2}}+\cdots+\phi_{i_{k-1}} .
$$

We make the latest cocycle $\phi_{u}$ to be dependent on others. In other words, the cocycle class $[\phi]$ which is born at the time $i+1$ is chosen to be killed at time when $b_{u}$ was introduced. This pairing matches that of the standard persistence algorithm where the youngest basis element is always paired first. We add the vector $\mathrm{a}_{\partial \sigma}$ to all annotations of $(p-1)$-simplices whose $u$ th element is nonzero. This zeroes out the $u$ th element of all annotations of $(p-1)$-simplices. We simply delete this element from all such annotations. See Figure 3(b).

Notice that determining if we have case (i) or (ii) can be done easily in $O(p g)$ time by checking the annotation of $\partial \sigma$. Indeed, this is achieved because the annotation already localizes the co-homology basis to each individual simplex.

\subsection{Elementary collapse}

The case for handling collapse is more interesting. It has three distinct steps, (i) elementary inclusions to satisfy the so called link condition, (ii) local annotation transfer to prepare for the collapse, and (iii) collapse of the simplices with updated annotations. We explain each of these steps now. 
The elementary inclusions that may precede the final collapse are motivated by a result that connects collapses with the change in (co)homology. Consider an elementary collapse $K_{i} \stackrel{f_{i}}{\rightarrow} K_{i+1}$ where the vertex pair $(u, v)$ collapses to $u$. The following link condition, introduced in [15] and later used to preserve homotopy [1], becomes relevant.

Definition 4.1 A vertex pair $(u, v)$ in a simplicial complex $K_{i}$ satisfies the link condition if the edge $\{u, v\} \in$ $K_{i}$, and $\mathrm{L} k u \cap \mathrm{L} k v=\mathrm{L} k\{u, v\}$. An elementary collapse $f_{i}: K_{i} \rightarrow K_{i+1}$ satisfies the link condition if the vertex pair on which $f_{i}$ is not injective satisfies the link condition.
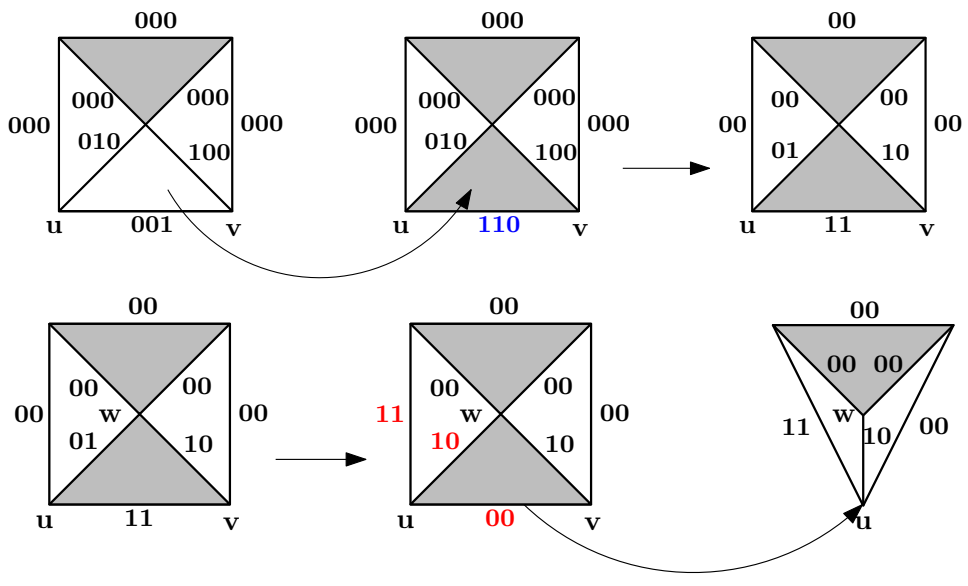

Figure 4: Annotation updates for elementary collapse: inclusion of a triangle to satisfy the link condition (upper row), annotation transfer and actual collapse (lower row); annotation 11 of the vanishing edge $u v$ is added to all edges (cofaces) adjoining $u$.

Proposition 4.2 [1] If an elementary collapse $f_{i}: K_{i} \rightarrow K_{i+1}$ satisfies the link condition, then the underlying spaces $\left|K_{i}\right|$ and $\left|K_{i+1}\right|$ remain homotopy equivalent and hence the induced homomorphisms $f_{i_{*}}: H_{*}\left(K_{i}\right) \rightarrow H_{*}\left(K_{i+1}\right)$ and $f_{i}^{*}: H^{*}\left(K_{i}\right) \leftarrow H^{*}\left(K_{i+1}\right)$ are isomorphisms.

If an elementary collapse satisfies the link condition, we can perform the col$\left.K_{i} \stackrel{f_{i}}{\longrightarrow}\right|_{\hat{K}_{i}} K_{i+1}^{\prime}$ lapse knowing that the (co)homology does not change. Otherwise, we know that the (co)homology is affected by the collapse and it should be reflected in our updates for annotations. The diagram at the left provides a precise means to carry out the change in (co)homology. Let $S$ be the set of simplices in non-decreasing order of dimensions, whose absence from $K_{i}$ makes $(u, v)$ violate the link condition. For each such simplex $\sigma \in S$, we modify the annotations of every simplex which we would have done if $\sigma$ were to be inserted. Thereafter, we carry out the rest of the elementary collapse. In essence, implicitly, we obtain an intermediate complex $\hat{K}_{i}=K_{i} \cup S$ where the diagram on the left commutes. Here, $f_{i}^{\prime}$ is induced by the same vertex map that induces $f_{i}$, and $j$ is an inclusion. This means that the persistence of $f_{i}$ is identical to that of $f_{i}^{\prime} \circ j$ which justifies our action of elementary inclusions followed by the actual collapses.

We remark that this is the only place where we may insert implicitly a simplex $\sigma$ in the current approach. The number of such $\sigma$ is usually much smaller than the number of simplices in the cone $u * \overline{\mathrm{St} v}$ that we would need to insert for the algorithm using coning.

Next, we transfer annotations in $\hat{K}_{i}$. This step locally changes the annotations for simplices containing the vertices $u$ and/or $v$. The following definition facilitates the description. 
Definition 4.3 For an elementary collapse $f_{i}: K_{i} \rightarrow K_{i+1}$, a simplex $\sigma \in K_{i}$ is called vanishing if the cardinality of $f_{i}(\sigma)$ is one less than that of $\sigma$. Two simplices $\sigma$ and $\sigma^{\prime}$ are called mirror pairs if one contains $u$ and the other $v$, and share rest of the vertices. In Figure 4 lower row), the vanishing simplices are $\{\{u, v\},\{u, v, w\}\}$ and the mirror pairs are $\{\{u\},\{v\}\},\{\{u, w\},\{v, w\}\}$.

In an elementary collapse that sends $(u, v)$ to $u$, all vanishing simplices need to be deleted, and all simplices containing $v$ need to be pulled to the vertex $u$ (which are their mirror partners). We update the annotations in such a way that the annotations of all vanishing simplices become zero, and those of each pair of mirror simplices become the same. Once this is achieved, the collapse is implemented by simply deleting the vanishing simplices and replacing $v$ with $u$ in all simplices containing $v$ without changing their annotations. The following proposition provides the justification behind the specific update operators that we perform.

Proposition 4.4 Let $K$ be a simplicial complex and a : $K(p) \rightarrow \mathbb{Z}_{2}^{g}$ be a valid annotation. Let $\sigma \in K(p)$ be any $p$-simplex and $\tau$ any of its $(p-1)$-faces. Adding $\mathrm{a}_{\sigma}$ to the annotation of all cofaces of $\tau$ of codimension 1 produces a valid annotation for $K(p)$. Furthermore, the cohomology basis corresponding to the annotations remains unchanged by this modification.

Proof: Let $\left\{\left[\phi_{1}\right], \ldots,\left[\phi_{g}\right]\right\}$ be a cohomology basis of $H^{p}(K)$ corresponding to a : $K(p) \rightarrow \mathbb{Z}_{2}^{g}$ as stated in Proposition 3.3. Let $T$ be the set of cofaces of $\tau$ of codimension 1 and

$$
\phi_{i}^{\prime}\left(\sigma^{\prime}\right)= \begin{cases}\phi_{i}\left(\sigma^{\prime}\right) & \text { if } \sigma^{\prime} \in K(p) \backslash T \\ \phi_{i}\left(\sigma^{\prime}\right)+\phi_{i}(\sigma) & \text { if } \sigma^{\prime} \in T\end{cases}
$$

By construction, $\phi_{i}^{\prime}$ is the cochain that corresponds to the new annotation obtained by adding $\mathrm{a}_{\sigma}$ to that of the simplices in $T$. We prove that $\phi_{i}^{\prime}$ is a cocycle in the class $\left[\phi_{i}\right]$. Therefore, $\left\{\left[\phi_{1}^{\prime}\right], \ldots,\left[\phi_{g}^{\prime}\right]\right\}$ is a cohomology basis of $H^{p}(K)$. The new annotation is valid by Proposition 3.3 and the cohomology bases remain unchanged.

If $\phi_{i}(\sigma)=0$, we have $\phi_{i}^{\prime}=\phi_{i}$ and thus $\left[\phi_{i}^{\prime}\right]=\left[\phi_{i}\right]$ trivially. So, assume that $\phi_{i}(\sigma)=1$. In this case $\phi_{i}^{\prime}=1+\phi_{i}$ on $T$ and equals $\phi_{i}$ everywhere else. Consider the $(p-1)$-cochain $\phi$ defined by $\phi(\tau)=1$ and $\phi\left(\tau^{\prime}\right)=0$ for every $\tau^{\prime} \in K_{p-1} \backslash \tau$. Then the coboundary $\delta \phi$ is a $p$-cochain that is 1 for every simplex in $T$ and 0 on other $p$-simplices. We can write $\phi_{i}^{\prime}=\phi_{i}+\delta \phi$. It follows that $\left[\phi_{i}^{\prime}\right]=\left[\phi_{i}\right]$.

Consider an elementary collapse $f_{i}: K_{i} \rightarrow K_{i+1}$ that sends $(u, v)$ to $u$. We update the annotations in $K_{i}$ as follows. First, note that the vanishing simplices are exactly those simplices containing the edge $\{u, v\}$. For every $p$-simplex containing $\{u, v\}$, i.e., a vanishing simplex, exactly two among its $(p-1)$-faces are mirror simplices, and all other remaining $(p-1)$-faces are vanishing simplices. Let $\sigma$ be a vanishing $p$-simplex and $\tau$ be its $(p-1)$-face that is a mirror simplex containing $u$. We add $\mathrm{a}_{\sigma}$ to the annotations of all cofaces of $\tau$ of codimension 1 including $\sigma$. We call this an annotation transfer for $\sigma$. By Proposition 4.4 the new annotation generated by this process corresponds to the old cohomology basis for $K_{i}$. This new annotation has $\mathrm{a}_{\sigma}$ as zero since $\mathrm{a}_{\sigma}+\mathrm{a}_{\sigma}=0$. See the the lower row of Figure 4

We perform the above operation for each vanishing simplex. It turns out that by using the relations of vanishing simplices and mirror simplices, each mirror simplex eventually acquires an identical annotation to that of its partner. Specifically, we have the following observation.

Proposition 4.5 After all possible annotation transfers involved in a collapse, (i) each vanishing simplex has a zero annotation; and (ii) each mirror simplex $\tau$ has the same annotation as its mirror partner simplex $\tau^{\prime}$. 
Proof: Our algorithm performs an annotation transfer for every vanishing simplex. Furthermore, the annotation transfer for a vanishing simplex $\sigma$ does not affect the annotation of any other vanishing simplex. Hence, the annotation of each vanishing simplex $\sigma$ is updated exactly once after which it becomes zero and remains so throughout the rest of the annotation transfers for other vanishing simplices. This proves claim (i).

For claim (ii), consider a pair of $(p-1)$-dimensional mirror simplices $\tau=\left\{u, u_{2}, \ldots, u_{p}\right\}$ and $\tau^{\prime}=$ $\left\{v, u_{2}, \ldots, u_{p}\right\}$. Since $(u, v)$ satisfies the link condition, it is necessary that the $p$-simplex $\alpha=\left\{u, v, u_{2}, \ldots, u_{p}\right\}$ must exist in $\hat{K}_{i}$. Thus, we have that $\mathrm{a}_{\partial \alpha}=0$. On the other hand, other than $\tau$ and $\tau^{\prime}$, any $(p-1)$-face of $\alpha$ is a vanishing simplex, and by Claim (i), in the end, has zero annotation. Therefore, after all annotation transfers, $a_{\partial \alpha}=a_{\tau}+a_{\tau^{\prime}}=0$, implying that $a_{\tau}=a_{\tau^{\prime}}$.

Subsequent to the annotation transfer, the annotation of $\hat{K}_{i}$ fits for actual collapse since each pair of mirror simplices which are collapsed to a single simplex get the identical annotation and the vanishing simplex acquires the zero annotation. Furthermore, Proposition 4.4 tells us that the cohomology basis does not change by annotation transfer which aligns with the fact that $f_{i}^{\prime *}: H^{*}\left(\hat{K}_{i}\right) \leftarrow H^{*}\left(K_{i+1}\right)$ is indeed an isomorphism. Accordingly, no time stamp changes after the annotation transfer and the actual collapse. The next section presents formal statements justifying the algorithm for annotation updates.

\section{Justification}

In this section we justify the algorithm for annotation updates. Generically assume $f: K \rightarrow K^{\prime}$ is an elementary map inducing a homomorphism $H^{p}(K) \stackrel{f^{*}}{\leftarrow} H^{p}\left(K^{\prime}\right)$ in $\mathcal{M}^{*}$ where $K=K_{i}$ and $K^{\prime}=K_{i+1}$ for some $i \in\{1, \ldots, n\}$. Let $\left\{\phi_{i}^{\prime}\right\}$ be the cochains corresponding to the annotations computed for $K^{\prime}$ given a valid annotation $\left\{\phi_{i}\right\}$ for $K$. First, we show that the computed annotations remain valid (proof in Appendix $\mathbb{B}$, that is, $\left\{\phi_{i}^{\prime}\right\}$ indeed forms a cohomology basis for $H^{p}\left(K^{\prime}\right)$. Then, we show in Propositions 5.2 and 5.3 how the cohomology bases $\left\{\left[\phi_{i}^{\prime}\right]\right\}$ and $\left\{\left[\phi_{i}\right]\right\}$ for $K^{\prime}$ and $K$ respectively correspond under the homomorphism $f^{*}$. The time stamps used by the algorithm concur with this correspondence.

Proposition 5.1 Let $\left\{\left[\phi_{i}\right]\right\}$ be the cohomology basis for $H^{p}(K)$ given by a valid annotation for $K$ and $\left\{\phi_{i}^{\prime}\right\}$ be the cochains corresponding to the annotation computed for $K^{\prime}$ by the update algorithm. Then, $\left\{\left[\phi_{i}^{\prime}\right]\right\}$ is a cohomology basis for $H^{p}\left(K^{\prime}\right)$.

First, we focus on when $f$ is an elementary inclusion. If $f$ is an elementary inclusion, it is known that in the persistence module, $f_{*}$ is either injective in which case a new class is born, or surjective in which case a class is killed. In the dual module with cohomology, $f^{*}$ switches the role, that is, $f^{*}$ is surjective when $f_{*}$ is injective and vice versa.

Proposition 5.2 Let $\sigma:=K^{\prime} \backslash K$ be a p-simplex inserted for inclusion $f: K \rightarrow K^{\prime}$.

i. $f_{*}$ is injective ( $f^{*}$ is surjective): Let $\left[\phi_{1}\right], \ldots,\left[\phi_{g}\right]$ be a basis of $H^{p}(K)$ given by a valid annotation. Let $\phi_{1}^{\prime}, \ldots, \phi_{g+1}^{\prime}$ be the cochains that correspond to the annotation computed for $K^{\prime}$ by the update algorithm. Then, $\left[\phi_{i}\right]=f^{*}\left(\left[\phi_{i}^{\prime}\right]\right)$ for $i=1, \ldots, g$ and $f^{*}\left(\left[\phi_{g+1}^{\prime}\right]\right)=0$. Cohomology bases for dimensions other than p remain unchanged.

ii. $f_{*}$ is surjective $\left(f^{*}\right.$ is injective): Let $\left\{\left[\phi_{1}\right], \ldots,\left[\phi_{g}\right]\right\}$ be a basis for $H^{p-1}$ given by a valid annotation. Let $\phi_{1}^{\prime}, \ldots, \phi_{g-1}^{\prime}$ be the cochains that correspond to the annotation computed for $K^{\prime}$ by the update algorithm which deletes the uth element. Then, for $1 \leq i<u,\left[\phi_{i}\right]=f^{*}\left(\left[\phi_{i}^{\prime}\right]\right)$ and for $i \geq u$, $\left[\phi_{i+1}\right]=f^{*}\left(\left[\phi_{i}^{\prime}\right]\right)$. Cohomology bases for dimensions other than $p-1$ remain unchanged. 
Proof: We provide the proof for (i) here and defer the proof of (ii) to the appendix. Recall that $\sigma=$ $K^{\prime} \backslash K$ is a $p$-simplex inserted for inclusion. We observe that when $f$ is an inclusion, we have $f_{\#}(z)=z$ for any cycle $z$ in $K$ where $f_{\#}$ denotes the chain map induced by $f$.

Consider the case for (i). In this case, $\sigma$ creates a new $p$-cycle and no other $k$-cycle for $k \neq p$. The annotations for $k$-simplices for $k \neq p$ are not changed. Therefore, a basis for $H^{k}(K)$ for $k \neq p$ remains so in $H^{k}\left(K^{\prime}\right)$. So, we can focus only on the case $k=p$. The algorithm updates the annotations of $p$-simplices in $K$ by appending a $0 \in \mathbb{Z}_{2}$ for everyone except the simplex $\sigma$ which gets a $1 \in \mathbb{Z}_{2}$. The definition of the homomorphism $H^{p}(K) \stackrel{f^{*}}{\leftarrow} H^{p}\left(K^{\prime}\right)$ provides that, for every $i \in\{1, \ldots, g\}$, there is a cocycle $\phi$ defined by the homomorphism $z \mapsto \phi_{i}^{\prime}\left(f_{\#}(z)\right)$ where $f^{*}\left(\left[\phi_{i}^{\prime}\right]\right)=[\phi]$. If $\left[z_{1}\right], \ldots,\left[z_{g}\right]$ is a basis of the homology group $H_{p}(K)$, the class $[\phi]$ is uniquely determined by the vector $\left[\phi\left(z_{1}\right), \ldots, \phi\left(z_{g}\right)\right]$. We have

$$
\begin{aligned}
{\left[\phi\left(z_{1}\right), \ldots, \phi\left(z_{g}\right)\right] } & =\left[\phi_{i}^{\prime}\left(f_{\#}\left(z_{1}\right), \ldots, \phi_{i}^{\prime}\left(f_{\#}\left(z_{g}\right)\right)\right]\right. \\
& =\left[\phi_{i}^{\prime}\left(z_{1}\right), \ldots, \phi_{i}^{\prime}\left(z_{g}\right)\right] \\
& =\left[\phi_{i}\left(z_{1}\right), \ldots, \phi_{i}\left(z_{g}\right)\right] .
\end{aligned}
$$

The last equality follows from the fact that $\phi_{i}^{\prime}\left(z_{j}\right)=\phi_{i}\left(z_{j}\right)$ because the $i$ th element in the annotation for $p$-simplices remains the same for $1 \leq i \leq g$. Since $\phi$ and $\phi_{i}$ evaluate the basis $\left[z_{1}\right], \ldots,\left[z_{g}\right]$ the same, we have $[\phi]=\left[\phi_{i}\right]$, that is, $f^{*}\left(\left[\phi_{i}^{\prime}\right]\right)=\left[\phi_{i}\right]$ as we are required to prove. Following the same argument we see that $\left[\phi_{g+1}^{\prime}\left(f_{\#}\left(z_{1}\right), \ldots, \phi_{g+1}^{\prime}\left(f_{\#}\left(z_{g}\right)\right)\right]=[0, \ldots, 0]\right.$ since the cycles $z_{i}$ for $i=1, \ldots, g$ do not include the simplex $\sigma$ and thus have the element 0 in the $(g+1)$-th position of the annotation for every simplex in them. Clearly, $f^{*}\left(\left[\phi_{g+1}^{\prime}\right]\right)=0$.

Similar to the case above, one can prove for case (ii) (see Appendix $B$ ) that

$$
\left[\phi\left(z_{1}\right), \ldots, \phi\left(z_{g}\right)\right]=\left[\phi_{i}\left(z_{1}\right), \ldots, \phi_{i}\left(z_{g}\right)\right]
$$

giving $f^{*}\left(\left[\phi_{i}^{\prime}\right]\right)=[\phi]=\left[\phi_{i}\right]$ for $i=\{1, \ldots, u-1\}$. The case for $i \in\{u+1, \ldots, g\}$ can be proved similarly. The only caveat is that the $u$ th element is zeroed out in annotation, so there is a left shift of the elements lying to the right of the $u$ th element in the annotation which accounts for the assertion $f^{*}\left(\left[\phi_{i}^{\prime}\right]\right)=\left[\phi_{i+1}\right]$.

Next, we consider the case when $f=f_{i}$ is an elementary collapse. Recall that we implement such a collapse as a composition of elementary inclusions $j$ and a vertex collapse $f^{\prime}$ where $f=f^{\prime} \circ j$. This induces the following sequence $H^{*}(K) \stackrel{j^{*}}{\leftarrow} H^{*}(\hat{K}) \stackrel{f^{\prime *}}{\leftarrow} H^{*}\left(K^{\prime}\right)$. Since we have already argued about inclusions, we only need to show that the annotation updates reflect the map $f^{\prime *}$.

Proposition 5.3 Let $\left[\phi_{1}\right], \ldots,\left[\phi_{g}\right]$ be a basis of $H^{p}(\hat{K})$ given by a valid annotation. Let $\phi_{1}^{\prime}, \ldots, \phi_{g}^{\prime}$ be the cochains that correspond to the annotation computed for $K^{\prime}$ by the update algorithm. Then, $\left[\phi_{i}\right]=f^{\prime *}\left(\left[\phi_{i}^{\prime}\right]\right)$ for $i=1, \ldots, g$.

Proof: First, recall that $f_{*}^{\prime}$ and hence $f^{\prime *}$ is an isomorphism due to Proposition 4.2 as the vertex pair $(u, v)$ satisfies the link condition in $\hat{K}$. Let $\left[z_{1}\right], \ldots,\left[z_{g}\right]$ be a basis in $H_{*}(\hat{K})$. As before, let $\phi$ be a cocycle defined by the homomorphism $z \mapsto \phi_{i}^{\prime}\left(f_{\#}^{\prime}(z)\right)$ where $f^{\prime *}\left(\left[\phi_{i}^{\prime}\right]\right)=[\phi]$. We have $\left[\phi\left(z_{1}\right), \ldots, \phi\left(z_{g}\right)\right]=$ $\left[\phi_{i}^{\prime}\left(f_{\#}^{\prime}\left(z_{1}\right)\right), \ldots, \phi_{i}^{\prime}\left(f_{\#}^{\prime}\left(z_{g}\right)\right)\right]$. Recall that we first carry out an annotation transfer in $\hat{K}$ to match the annotations for the mirror simplices and to zero out the annotations for the vanishing simplices. This update does not change the cohomology classes thanks to Proposition 4.4. So, we focus on the update due to the vertex collapse. Every pair of mirror simplices carries their annotation into the collapsed simplex, and vanishing simplices lose their zero annotations as they disappear. In effect, we have $\phi_{i}^{\prime}\left(f_{\#}^{\prime}\left(z_{j}\right)\right)=\phi_{i}\left(z_{j}\right)$, giving us that $\left[\phi\left(z_{1}\right), \ldots, \phi\left(z_{g}\right)\right]=\left[\phi_{i}\left(z_{1}\right), \ldots, \phi_{i}\left(z_{g}\right)\right]$. Therefore, $f^{\prime *}\left(\left[\phi_{i}^{\prime}\right]\right)=[\phi]=\left[\phi_{i}\right]$ for $i=1, \ldots, g$. 


\section{Application to topological data analysis}

In topological data analysis, several applications and approaches use Rips complex filtration [1, 14, 24]. The computation of the persistence diagram or its approximation for a Rips filtration appears to be a key step in these applications. However, the size of this filtration becomes a bottleneck because of the inclusive nature of Rips complexes. A natural way to handle this problem is to successively subsample the input data and build a filtration on top of them. We show how one can apply our results from previous sections to approximate the persistence diagrams of a Rips filtration from such a sparser filtration. Given a set of points $V \subset \mathbb{R}^{d}$ (Similar to [24], results in this section can be extended to any metric space with doubling dimension $d$.), let $\mathcal{R}^{r}(V)$ denote the Rips complex on the point set $V$ with parameter $r$. That is, a $k$-simplex $\sigma=\left\{u_{0}, \ldots, u_{k}\right\} \subseteq V$ is in $\mathcal{R}^{r}(V)$ if and only if $\left\|u_{i}-u_{j}\right\| \leq r$ for any $i, j \in[0, k]$. We present an algorithm to approximate the persistence diagram for the following Rips filtration. The parameters $\alpha>0$ and $0 \leq \varepsilon \leq 1$ are assumed to be preselected.

$$
\mathcal{R}^{\alpha}(V) \hookrightarrow \mathcal{R}^{(1+\varepsilon) \alpha}(V) \cdots \hookrightarrow \mathcal{R}^{(1+\varepsilon)^{m} \alpha}(V) .
$$

The number of $k$-simplices in a Rips complex with $n$ vertices can be $\Theta\left(n^{k+1}\right)$. This makes computing the persistent homology of the above filtration costly. In [24], Sheehy proposed to approximate the persistence diagram of the above filtration by another Rips filtration where each simplicial complex involved has size only linear in $n$. This approach allows vertices to be collapsed (deleted) with a weighting scheme when the parameter $r$ for the Rips complex becomes large, which helps to keep the size of the simplicial complex at each stage small.

In this section, we provide an alternative approach to approximate the persistence diagram of the filtration given in (1). We achieve sparsification by subsampling as in [24], but our persistence algorithm for simplicial maps allows us to handle the sequence of complexes induced by the clustering / collapsing of vertices directly instead of an additional weighting scheme. We consider two sparsification schemes, one produces a sequence of sparsified Rips complexes, and the other produces a sequence of graph induced complexes (GICs) which have been shown to be even sparser in practice [13]. Asymptotically, both sequences have sizes linear in number of vertices.

\subsection{Persistence diagram approximation by sparsified Rips complex}

Given a set of points $V$, we say that $V^{\prime} \subseteq V$ is a $\delta$-net of $V$ if (i) for any point $v \in V$, there exists a point $v^{\prime} \in V$ such that $\left\|v-v^{\prime}\right\| \leq \delta$; and (ii) no two points in $V^{\prime}$ are within $\delta$ distance. A $\delta$-net $V^{\prime}$ can be easily constructed by a standard greedy approach by taking furthest points iteratively or by more sophisticated and efficient methods as in [10, 21]).

Now set $V_{0}:=V$. We first construct a sequence of point sets $V_{k}, k=0,1, \ldots, m$, such that $V_{k+1}$ is a $\frac{\alpha \varepsilon^{2}}{2}(1+\varepsilon)^{k-1}$-net of $V_{k}$. Consider the following vertex maps $\pi_{k}: V_{k} \rightarrow V_{k+1}$, for $k \in[0, m-1]$, where for any $v \in V_{k}, \pi_{k}(v)$ is the vertex in $V_{k+1}$ that is closest to $v$. Define $\hat{\pi}_{k}: V_{0} \rightarrow V_{k+1}$ as $\hat{\pi}_{k}(v)=\pi_{k} \circ \cdots \pi_{0}(v)$.

Each vertex map $\pi_{k}$ induces a well-defined simplicial map $h_{k}: \mathcal{R}^{\alpha(1+\varepsilon)^{k}}\left(V_{k}\right) \rightarrow \mathcal{R}^{\alpha(1+\varepsilon)^{k+1}}\left(V_{k+1}\right)$. Indeed, since $V_{k+1}$ is a $\frac{1}{2} \alpha \varepsilon^{2}(1+\varepsilon)^{k-1}$-net of $V_{k}$, for each edge $e=\{u, v\}$ from $\mathcal{R}^{\alpha(1+\varepsilon)^{k}}\left(V_{k}\right)$, we have

$$
\begin{aligned}
\left\|\pi_{k}(u)-\pi_{k}(v)\right\| & \leq\|u-v\|+\|u-\pi(u)\|+\|v-\pi(v)\| \\
& \leq \alpha(1+\varepsilon)^{k}+\alpha \varepsilon^{2}(1+\varepsilon)^{k-1} \\
& \leq \alpha(1+\varepsilon)^{k+1} .
\end{aligned}
$$

Hence $\pi_{k}(u) \pi_{k}(v)$ is an edge in $\mathcal{R}^{\alpha(1+\varepsilon)^{k+1}}\left(V_{k+1}\right)$. Since in a Rips complex, higher dimensional simplices are determined by the edges, every simplex $\left\{u_{0}, \ldots, u_{d}\right\}$ in $\mathcal{R}^{\alpha(1+\varepsilon)^{k}}\left(V_{k}\right)$ has a well-defined image 
$\left\{\pi_{k}\left(u_{0}\right), \ldots, \pi_{k}\left(u_{d}\right)\right\}$ in $\mathcal{R}^{\alpha(1+\varepsilon)^{k+1}}\left(V_{k+1}\right)$. Hence, each $h_{k}$ is well-defined providing the filtration:

$$
\mathcal{R}^{\alpha}\left(V_{0}\right) \stackrel{h_{0}}{\longrightarrow} \mathcal{R}^{\alpha(1+\varepsilon)}\left(V_{1}\right) \cdots \stackrel{h_{m-1}}{\longrightarrow} \mathcal{R}^{\alpha(1+\varepsilon)^{m}}\left(V_{m}\right) .
$$

In other words, as the parameter $r=\alpha(1+\varepsilon)^{k}$ increases, we can simply consider the Rips complex built upon the sparsified data points $V_{k}$. Note that the sequence above is not connected by inclusion maps and thus classical persistent algorithms cannot be applied directly; while our algorithm from Section 4 can be used here in a straightforward manner.

Our main observation is that the persistence diagram of the sequence of simplicial maps in (2) approximates that of the inclusion maps in (1). In particular, we show that the persistence modules induced by these two sequences interleave in the sense described in [6].

First, we need maps to connect these two sequences. For this, we observe that the vertex map $\hat{\pi}_{k}$ : $V_{0} \rightarrow V_{k+1}$ also induces a simplicial map $\hat{h}_{k}: \mathcal{R}^{\alpha(1+\varepsilon)^{k}}\left(V_{0}\right) \rightarrow \mathcal{R}^{\alpha(1+\varepsilon)^{k+1}}\left(V_{k+1}\right)$. To establish that this simplicial map is well-defined, it can be shown that if there is an edge $\{u, v\}$ in $\mathcal{R}^{\alpha(1+\varepsilon)^{k}}\left(V_{0}\right)$, then there is an edge $\hat{\pi}_{k}(u) \hat{\pi}_{k}(v)$ in $\mathcal{R}^{\alpha(1+\varepsilon)^{k+1}}\left(V_{k+1}\right)$.

Claim 6.1 Each triangle in the following diagram commutes at the homology level.

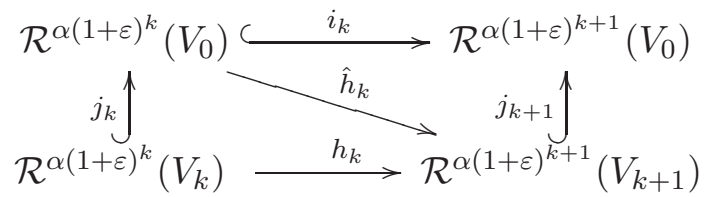

Here, the maps $i_{k} s$ and $j_{k} s$ are canonical inclusions. The simplicial maps $\hat{h}_{k}$ and $h_{k}$ are induced by the vertex maps $\hat{\pi}_{k}: V_{0} \rightarrow V_{k+1}$ and $\pi_{k}: V_{k} \rightarrow V_{k+1}$, respectively, as described before.

Proof: First, we consider the bottom triangle. Note that the vertex map $\hat{\pi}_{k}$ restricted on the set of vertices $V_{k}$ is the same as the vertex map $\pi_{k}$. (That is, for a vertex $u \in V_{k} \subseteq V_{0}, h_{k}(u)=\hat{h}_{k}(u)$.) Thus $h_{k}=\hat{h}_{k} \circ j_{k}$. Hence the bottom triangle commutes both at the simplicial complex level and at the homology level.

Consider the top triangle. We claim that the map $j_{k+1} \circ \hat{h}_{k}$ is contiguous to the inclusion $i_{k}$. Since two contiguous maps induce the same homomorphisms at the homology level, the top triangle commutes at the homology level.

This claim can be verified by the definition of contiguous maps. Given a simplex $\sigma \in \mathcal{R}^{\alpha(1+\varepsilon)^{k}}\left(V_{0}\right)$, we wish to show that vertices from $\sigma \cup \hat{h}_{k}(\sigma)$ span a simplex in $\mathcal{R}^{\alpha(1+\varepsilon)^{k+1}}\left(V_{0}\right)$. Since $\mathcal{R}^{\alpha(1+\varepsilon)^{k+1}}\left(V_{0}\right)$ is a Rips complex, we only need to show that for any two vertices $u$ and $v$ from $\sigma \cup \widehat{h}_{k}(\sigma)$, the edge $u v$ has length less than $\alpha(1+\varepsilon)^{k+1}$ (and thus in $\mathcal{R}^{\alpha(1+\varepsilon)^{k+1}}\left(V_{0}\right)$ ). If $u$ and $v$ are both from $\sigma$ or both from $\hat{h}_{k}(\sigma)$, then obviously $\|u-v\| \leq \alpha(1+\varepsilon)^{k+1}$. Otherwise, assume without loss of generality that $v \in \sigma$ and $u \in \hat{h}_{k}(\sigma)$ where $u=\hat{\pi}_{k}(\bar{u})$ for some $\bar{u} \in \sigma$. It then follows that,

$$
\begin{aligned}
\|u-v\| & \leq\|u-\bar{u}\|+\|\bar{u}-v\| \\
& \leq \frac{\alpha \varepsilon^{2}}{2(1+\varepsilon)} \sum_{i=0}^{k}(1+\varepsilon)^{i}+\alpha(1+\varepsilon)^{k}<\alpha(1+\varepsilon)^{k+1} .
\end{aligned}
$$

Therefore, the vertices of $\sigma \cup \widehat{h}_{k}(\sigma)$ span a simplex in $\mathcal{R}^{\alpha(1+\varepsilon)^{k+1}}\left(V_{0}\right)$.

The above claim implies that the persistence modules induced by sequences (1) and (2) are weakly $\frac{\log (1+\varepsilon)}{2}$-interleaved at the log-scale. By Theorem 4.3 of [6], we thus conclude with the following: 
Proposition 6.2 The persistence diagram of the sequence (2) provides a $\frac{3 \log (1+\varepsilon)}{2}$-approximation of the persistence diagram of the sequence (11) at the log-scale.

Finally, since $V_{k+1}$ is a $\delta$-net of $V_{k}$ for $\delta=\frac{\alpha \varepsilon^{2}}{2}(1+\varepsilon)^{k-1}$, we can show by a standard packing argument that each $\mathcal{R}^{\alpha(1+\varepsilon)^{k}}\left(V_{k}\right)$ is of size linear in $n$. See Proposition C. 1 in appendix. Note that the persistence diagram of the simplicial maps in (2) can be computed by our algorithm in Section 4 Putting everything together, we have the following result.

Theorem 6.3 Given a set of $n$ points $V$ in a metric space with doubling-dimension $d$ and $0 \leq \varepsilon \leq 1$, we can $\frac{3 \log (1+\varepsilon)}{2}$-approximate the persistence diagram of the Rips complex filtration (1) by that of the filtration (21). The p-skeleton of each simplicial complex involved in (2) has size $O\left(\left(\frac{1}{\varepsilon}\right)^{O(d p)} n\right)$.

\subsection{Persistence diagram approximation by graph induced complex}

We now present an alternative way to construct a sequence of complexes for gradually sparsified or subsampled points. The graph induced complex (GIC) proposed in [13] works on a subsample as the sparsified Rips complex does. However, it contains much fewer simplices in practice. In [13], it was shown how GIC can be used to estimate the homology of compact sets by investigating the persistence of a single simplicial map. Here we show how one can build a sequence of GICs to approximate the persistence diagram of a Rips filtration. Similar to the case of a sequence of sparsified Rips complexes, simplicial maps occur naturally to connect these GICs in the sequence.

Definition 6.4 Let $G(V)$ be a graph with the vertex set $V$ and let $\nu: V \rightarrow V^{\prime}$ be a vertex map where $\nu(V)=V^{\prime} \subseteq V$ is a subset of vertices. The graph induced complex $\mathcal{G}\left(V, V^{\prime}, \nu\right):=\mathcal{G}\left(G(V), V^{\prime}, \nu\right)$ is defined as the simplicial complex where a $k$-simplex $\sigma=\left\{v_{1}^{\prime}, v_{2}^{\prime}, \ldots, v_{k+1}^{\prime}\right\}$ is in $\mathcal{G}\left(V, V^{\prime}, \nu\right)$ if and only if there exists a $(k+1)$-clique $\left\{v_{1}, v_{2}, \ldots, v_{k+1}\right\} \subseteq V$ so that $\nu\left(v_{i}\right)=v_{i}^{\prime}$ for each $i \in\{1,2, \ldots, k+1\}$. To see that it is indeed a simplicial complex, observe that a subset of a clique is also a clique. Let $G(V)$ be called the base-graph for $\mathcal{G}\left(V, V^{\prime}, \nu\right)$.

Intuitively, the vertex map $\nu$ maps a cluster of vertices from $V$ to a single vertex $v^{\prime} \in V^{\prime}$, and these vertices constitute the "Voronoi cell" of the site $v^{\prime}$. The GIC $\mathcal{G}\left(V, V^{\prime}, \nu\right)$ is somewhat the combinatorial dual of such a Voronoi diagram. In our case the base graph $G(V)$ is the 1-skeleton of the Rips complex $\mathcal{R}^{r}\left(V_{0}\right)$ and the vertex map $\nu$ is the map $\hat{\pi}_{k}: V_{0} \rightarrow V_{k+1}$ as defined in the last section. Denote $\mathcal{G}^{r}\left(V_{0}, V_{k}\right):=$ $\mathcal{G}\left(V_{0}, V_{k}, \hat{\pi}_{k-1}\right)$ constructed using the 1-skeleton of $\mathcal{R}^{r}\left(V_{0}\right)$ as the base-graph. It is easy to show that by the definition of $\hat{\pi}_{k}$ and construction of $V_{k} \mathrm{~s}$, the vertex map $\pi_{k}: V_{k} \rightarrow V_{k+1}$ induces a well-defined simplicial map $f_{k}: \mathcal{G}^{\alpha(1+\varepsilon)^{k-1}}\left(V_{0}, V_{k}\right) \longrightarrow \mathcal{G}^{\alpha(1+\varepsilon)^{k}}\left(V_{0}, V_{k+1}\right)$, giving rise to the following sequence:

$$
\begin{aligned}
\mathcal{G}^{\alpha}\left(V_{0}, V_{1}\right) \stackrel{f_{1}}{\longrightarrow} \mathcal{G}^{\alpha(1+\varepsilon)}\left(V_{0}, V_{2}\right) \stackrel{f_{2}}{\longrightarrow} \mathcal{G}^{\alpha(1+\varepsilon)^{2}}\left(V_{0}, V_{3}\right) \cdots \\
\stackrel{f_{m-1}}{\longrightarrow} \mathcal{G}^{\alpha(1+\varepsilon)^{m-1}}\left(V_{0}, V_{m}\right) .
\end{aligned}
$$

We prove that the persistence diagram of the above filtration induced by simplicial maps $f_{k}$ 's has the same approximation factor to the persistence of diagram of the filtration (1) as that of the filtration (2). Thus, we have:

Theorem 6.5 Given a set of $n$ points $V$ in a metric space with doubling-dimension $d$ and $0 \leq \varepsilon \leq 1$, we can $\frac{3 \log (1+\varepsilon)}{2}$-approximate the persistence diagram of the Rips complex filtration (1) by that of the filtration (3). The p-skeleton of each simplicial complex involved in (3) has size $O\left(\left(\frac{1}{\varepsilon}\right)^{O(d p)} n\right)$. 
Proof: In sequence (3), $V_{k+1}$ is a $\delta_{k+1}$-net of $V_{k}$ for $\delta_{k+1}=\frac{\alpha \varepsilon^{2}}{2}(1+\varepsilon)^{k-1}(k=0,1, \ldots, m-1)$ as in the sequence of (2). Now consider $\hat{\pi}_{k}: V_{0} \rightarrow V_{k+1}$. It is immediate that $\left|p \hat{\pi}_{k}(p)\right| \leq \frac{\alpha \varepsilon^{2}}{2(1+\varepsilon)} \sum_{i=0}^{k}(1+$ $\varepsilon)^{i} \leq \frac{\alpha \varepsilon}{2}(1+\varepsilon)^{k}$ for each $p \in V_{0}$. In other words, $V_{k+1}$ is a $\frac{\alpha \varepsilon}{2}(1+\varepsilon)^{k}$-sample of $V_{0}$. Recall the GIC $\mathcal{G}^{\alpha(1+\varepsilon)^{k}}\left(V_{0}, V_{k+1}\right)$ is constructed based on the 1-skeleton of $\mathcal{R}^{\alpha(1+\varepsilon)^{k}}\left(V_{0}\right)$ (used as the base-graph). It is easy to show that $\hat{\pi}_{k}(p)$ induces a simplicial map $\hat{f}_{k}: \mathcal{R}^{\alpha(1+\varepsilon)^{k}}\left(V_{0}\right) \rightarrow \mathcal{G}^{\alpha(1+\varepsilon)^{k}}\left(V_{0}, V_{k+1}\right)$. To prove that the persistence diagram of the sequence (11) is approximated by that of the sequence (3), it is sufficient to show that the sequence (1) interleaves with the sequence (3). The following claim reveals the desired interleaving property. Its proof is similar to that of the Claim 6.1

Claim 6.6 Each triangle in the following diagram commutes at the homology level.

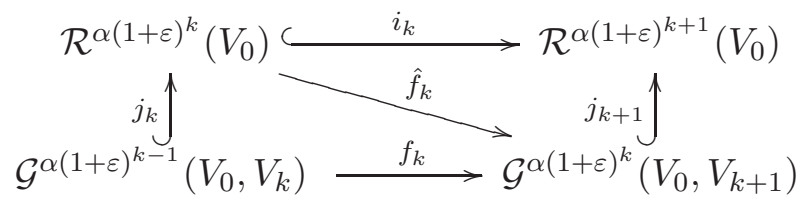

Here, the maps $i_{k} s$ and $j_{k} s$ are canonical inclusions. The simplicial map $\hat{f}_{k}$ is induced by the vertex map $\hat{\pi}_{k}: V_{0} \rightarrow V_{k+1}$, and the simplicial map $f_{k}=\hat{f}_{k} \circ j_{k}$.

Note that for every edge $u v$ in $\mathcal{G}^{\alpha(1+\varepsilon)^{k}}\left(V_{0}, V_{k+1}\right)$, there is an edge $a b$ in $\mathcal{R}^{\alpha}(1+\varepsilon)^{k}\left(V_{0}\right)$ such that $\hat{\pi}_{k}(a)=u$ and $\hat{\pi}_{k}(b)=v$. Since $V_{k+1}$ is a $\frac{\alpha \varepsilon}{2}(1+\varepsilon)^{k}$-sample of $V_{0}$, one has that

$$
|u v| \leq|u a|+|v b|+|a b| \leq \alpha \varepsilon(1+\varepsilon)^{k}+\alpha(1+\varepsilon)^{k}=\alpha(1+\varepsilon)^{k+1} .
$$

Therefore, the 1-skeleton of the graph induced complex $\mathcal{G}^{\alpha(1+\varepsilon)^{k}}\left(V_{0}, V_{k+1}\right)$ is a subcomplex of the 1skeleton of $\mathcal{R}^{\alpha(1+\varepsilon)^{k+1}}\left(V_{k+1}\right)$. Consequently, $\mathcal{G}^{\alpha(1+\varepsilon)^{k}}\left(V_{0}, V_{k+1}\right)$ is a subcomplex of $\mathcal{R}^{\alpha(1+\varepsilon)^{k+1}}\left(V_{k+1}\right)$ which is the maximal simplicial complex containing its 1-skeleton. This observation implies that the sequence (3) has smaller size compared to the sequence (2). Furthermore, although the asymptotic space complexity of each GIC is the same as that of the sparsified Rips complex, in practice, the size of GICs can be far smaller; see [13]. However, the construction of each GIC is more expensive, as one needs to compute each $\mathcal{G}^{\alpha(1+\varepsilon)^{k}}\left(V_{0}, V_{k+1}\right)$ from the Rips complex $\mathcal{R}^{\alpha(1+\varepsilon)^{k}}\left(V_{0}\right)$ built on the original vertex set $V_{0}$, instead of the vertex set from the previous complex $\mathcal{G}^{\alpha(1+\varepsilon)^{k-1}}\left(V_{0}, V_{k}\right)$. Hence there is a trade-off of space versus time for the approaches given in Section 6.1

\section{Conclusions}

In this paper, we studied algorithms to compute the persistence diagram of a (monotone) filtration connected by simplicial maps efficiently. As discussed in [17], the algorithm based on the cohomology view in [16] has a good practical performance for the case of computing inclusion-induced non-zigzag persistence. Our annotation-based algorithm extends such a view of maintaining an appropriate cohomology basis to the case of vertex collapses. This allows us to compute the persistence diagram for a filtration connected by simplicial maps directly and efficiently. The coning approach in Section 2.3 works for any finite fields though the collapse based algorithm in Section 4 currently works with $\mathbb{Z}_{2}$ coefficients only. Although inclusions can be handled under other finite field coefficients, it is not clear how to handle collapses efficiently.

We believe that, as the scope of topological data analysis continues to broaden, further applications based on simplicial maps will arise. Currently, an efficient implementation of the persistence algorithm 
taking advantage of the compressed representation of annotations has been reported in [2]. We have also developed an efficient implementation of the persistence algorithms for simplicial maps in the same vein. The software named SimpPers is available from authors' web-pages.

\section{Acknowledgment}

We acknowledge the helpful comments of the reviewers and the support of the NSF grants CCF 1116258, CCF 1064416, CCF 1318595, and CCF 1319406.

\section{References}

[1] D. Attali, A. Lieutier, and D. Salinas. Efficient data structure for representing and simplifying simplicial complexes in high dimensions. Proc. 27th Annu. Sympos. Comput. Geom. (2011), 501-509.

[2] J.-D. Boissonnat, T. K. Dey, and C. Maria. The compressed annotation matrix: An efficient data structure for computing persistent cohomology. Proc. European Sympos. Algorithms (2013), Vol. 8125 Lecture Notes in Comput. Sci., 695-706.

[3] D. Burghelea and T. K. Dey. Topological persistence for circle-valued maps. Discrete Comput. Geom., 50(1) (2013), 69-98.

[4] O. Busaryev, S. Cabello, C. Chen, T. K. Dey, and Y. Wang. Annotating simplices with a homology basis and its applications. Proc. 13th Scandinavian Sympos. Workshops Algorithm Theory (SWAT 2012), (2012), 189-200.

[5] G. Carlsson. Topology and data. Bull. Amer. Math. Soc. 46 (2009), 255-308.

[6] F. Chazal, D. Cohen-Steiner, M. Glisse, L. J. Guibas, and S. Y. Oudot. Proximity of persistence modules and their diagrams. Proc. 25th Annu. Sympos. Comput. Geom. (2009), 237-246.

[7] C. Chen and M. Kerber. An output-sensitive algorithm for persistent homology. Proc. 27th Annu. Sympos. Comput. Geom. (2011), 207-216.

[8] G. Carlsson and V. de Silva. Zigzag persistence. Found. Comput. Math. 10 (4), 367-405, 2010.

[9] G. Carlsson, V. de Silva, and D. Morozov. Zigzag persistent homology and real-valued functions. Proc. 26th Annu. Sympos. Comput. Geom. (2009), 247-256.

[10] K. L. Clarkson. Nearest-Neighbor Searching and Metric Space Dimensions. In G. Shakhnarovich, T. Darrel, and P. Indyk, editors, Nearest-Neighbor Methods for Learning and Vision: Theory and Practice, (2006), 15-59.

[11] D. Cohen-Steiner, H. Edelsbrunner, and D. Morozov. Vines and vineyards by updating persistence in linear time. Proc. 22nd Annu. Sympos. Comput. Geom. (2006), 119-126.

[12] D. Cohen-Steiner, H. Edelsbrunner, and J. L. Harer. Stability of persistence diagrams. Discrete Comput. Geom. 37 (2007), 103-120.

[13] T. K. Dey, F. Fan, and Y. Wang. Graph Induced Complex on Point Data. Proc. 29th Annu. Sympos. Comput. Geom. (2013), 107-116. 
[14] T. K. Dey, J. Sun, and Y. Wang. Approximating cycles in a shortest basis of the first homology group from point data. Inverse Problems 27 (2011), 124004. doi:10.1088/0266-5611/27/12/124004.

[15] T. K. Dey, H. Edelsbrunner, S. Guha and D. Nekhayev. Topology preserving edge contraction. Publications de l' Institut Mathematique (Beograd) 60(80) (1999), 23-45.

[16] V. de Silva, D. Morozov, and M. Vejdemo-Johansson. Persistent cohomology and circular coordinates. Discrete Comput. Geom. 45 (4) (2011), 737-759.

[17] V. de Silva, D. Morozov, and M. Vejdemo-Johansson. Dualities in persistent (co)homology . Inverse Problems. 27 (12) (2011), 124003.

[18] H. Edelsbrunner and J. Harer. Computational Topology: An Introduction. Amer. Math. Soc., Providence, Rhode Island, 2009.

[19] H. Edelsbrunner, D. Letscher, and A. Zomorodian. Topological persistence and simplification. Discrete Comput. Geom. 28 (2002), 511-533.

[20] R. Ghrist. Barcodes: The persistent topology of data. Bull. Amer. Math. Soc. 45 (2008), 61-75.

[21] S. Har-Peled and M. Mendel. Fast construction of nets in low dimensional metrics, and their applications. SIAM Journal on Computing. 35(5) (2006), 1148-1184.

[22] A. Hatcher. Algebraic Topology. Cambridge U. Press, New York, 2002.

[23] N. Milosavljević, D. Morozov, and P. Škraba. Zigzag persistent homology in matrix multiplication time. Proc. 27th Annu. Sympos. Comput. Geom. (2011), 216-225.

[24] D. Sheehy. Linear-Size Approximations to the Vietoris-Rips Filtration. Proc. 28th. Annu. Sympos. Comput. Geom. (2012), 239-247.

[25] A. Zomorodian and G. Carlsson. Computing persistent homology. Discrete Comput. Geom. 33 (2005), 249-274.

\section{A Missing proof from Section 2}

Proof of Proposition 2.5. Consider the surjective simplicial map $f^{\prime}: K \rightarrow f(K)$ defined as $f^{\prime}(\sigma)=f(\sigma)$ for any $\sigma \in K$. Writing $V=V(K)$ and $V^{\prime}=V\left(K^{\prime}\right)$, we have $f_{V}^{\prime}=f_{V}$. The simplicial map $f: K \rightarrow K^{\prime}$ is a composition $i \circ f^{\prime}$, where $i: f(K) \hookrightarrow K^{\prime}$ is the canonical inclusion $f(K) \subseteq K^{\prime}$. Obviously, the inclusion $i$ can be easily decomposed into a sequence of elementary inclusions. We now show that $f^{\prime}$ can be decomposed into a sequence of elementary collapses.

Let $A:=\left\{v \in V^{\prime}|| f_{\mathrm{V}}^{-1}(v) \mid>1\right\}$. Hence $f_{\mathrm{V}}$ maps injectively onto $V^{\prime} \backslash A$. Order vertices in $A$ arbitrarily as $\left\{v_{1}, \ldots, v_{k}\right\}, k=|A|$, and let $A_{i}$ denote $f_{\mathrm{V}}{ }^{-1}\left(v_{i}\right)$. We now define $f_{i}$ and $K_{i}$ in increasing order of $i$. For the base case, set $K_{0}=K$. For any $i>0$, consider the vertex map $f_{\mathrm{A}_{i}}$ which is the injective map on $V\left(K_{i-1}\right) \backslash A_{i}$, but maps $A_{i}$ to $v_{i}$. We set $f_{i}$ to be the simplicial map induced by this vertex map $f_{\mathrm{A}_{i}}$, and set $K_{i}:=f_{i}\left(K_{i-1}\right)$. By construction, $f_{i}$ is a surjective simplicial map.

It is easy to see that the vertex map $f_{\mathrm{A}_{k}} \circ \cdots \circ f_{\mathrm{A}_{1}}$ equals $f_{\mathrm{V}}$. Hence, the induced simplicial map $f_{k} \circ \cdots \circ f_{1}: K \rightarrow K_{k}$ equals $f^{\prime}: K \rightarrow f(K)$. Furthermore, each $f_{i}$ can be decomposed into a sequence of elementary collapses, each induced by a vertex map that maps only two vertices from $A_{i}$ to $v_{i}$.

Proposition A.1 The simplicial maps $i^{\prime} \circ f$ and $i$ are contiguous. 
Proof: By definition of contiguous maps, we need to show that for any simplex $\sigma \in K, i(\sigma) \cup i^{\prime} \circ f(\sigma)$ is a simplex in $\hat{K}$. Note that $i(\sigma)=\sigma$.

First assume that the simplex $\sigma$ is not in St $v$. Since $f$ is an elementary collapse, we have $f(\sigma)=\sigma$ and $i^{\prime} \circ f(\sigma)=\sigma$. Hence, $i(\sigma) \cup\left(i^{\prime} \circ f\right)(\sigma)$ equals $\sigma$ which is also a simplex in $\hat{K}$.

Now assume that $\sigma \in \operatorname{St} v$, and $\sigma=\left\{u_{0}, \ldots, u_{d}\right\} \cup\{v\}$. Since $f(v)=u, f(\sigma)=\left\{u_{0}, \ldots, u_{d}\right\} \cup\{u\}$, and so is $\left(i^{\prime} \circ f\right)(\sigma)$. Hence the union of $i(\sigma)$ and $\left(i^{\prime} \circ f\right)(\sigma)$ is $B:=\left\{u_{0}, \ldots, u_{d}\right\} \cup\{u, u\}$, which is the simplex $\sigma \cup\{u\}$. On the other hand, by construction of $\hat{K}$, the simplex $\sigma \cup\{u\}$ is necessarily in $\hat{K}$. Hence $i(\sigma) \cup\left(i^{\prime} \circ f\right)(\sigma)$ is a simplex in $\hat{K}$ in this case too. Hence the maps $i$ and $i^{\prime} \circ f$ are contiguous.

Proposition A.2 $i_{*}^{\prime}: H_{*}\left(K^{\prime}\right) \rightarrow H_{*}(\hat{K})$ is an isomorphism.

Proof: Consider the projection map $\pi: \hat{K} \rightarrow K^{\prime}$ induced by the vertex map

$$
\pi(p)= \begin{cases}u & \text { if } p=v \\ p & \text { otherwise. }\end{cases}
$$

Let $i d_{\hat{K}}$ denote the identity map on $\hat{K}$. We show that: (i) $\pi$ is an elementary collapse, and (ii) the composition $i^{\prime} \circ \pi$ and $\mathrm{i} d_{\hat{K}}$ are contiguous. It is easy to see that $\pi \circ i^{\prime}$ is $\mathrm{i} d_{K^{\prime}}$. Then, $i^{\prime}$ is a (simplicial) homotopy equivalence and hence $i_{*}^{\prime}$ is an isomorphism.

Specifically, consider an arbitrary simplex $\sigma \in \hat{K}$. Let $\hat{X}=\operatorname{St}\{u, v\}$ be the star of $\{u, v\}$ in $\hat{K}$. If $\sigma \notin \hat{X}$, then by the construction of $\hat{K}, \sigma \in K^{\prime}$. In other words, $\pi(\sigma)=\sigma$ indeed exists in $K^{\prime}$ in this case. Furthermore, $i^{\prime} \circ \pi(\sigma)=\sigma$ and thus $\left(i^{\prime} \circ \pi\right)(\sigma) \cup \mathrm{i} d_{\hat{K}}(\sigma)=\sigma \in \hat{K}$.

Now consider the case $\sigma \in \hat{X}$, and assume that $\sigma=\left\{u_{0}, \ldots, u_{d}\right\} \cup A$ with $A \subseteq\{u, v\}$. To show that $\pi$ is well defined, we need to show that $\pi(\sigma)=\left\{u_{0}, \ldots, u_{d}, u\right\}$, is indeed a simplex in $K^{\prime}$.

(i) If $u \notin A$, then by the construction of $\hat{K}, \sigma$ has a pre-image in $K$ under the inclusion $i: K \rightarrow \hat{K}$. Hence $\sigma$ must also be a simplex in $K$, and under the map $f$, it is mapped to the simplex $\left\{u_{0}, \ldots, u_{d}, u\right\}$ in $K^{\prime}$. As such, $\pi(\sigma)$ exists in $K^{\prime}$ in this case. (ii) If $u \in A$, then the simplex $\sigma^{\prime \prime}=\left\{u_{0}, \ldots, u_{d}\right\} \cup(A \backslash\{u\})$ must exist in the closed star $\overline{\operatorname{St}_{K}\{u, v\}}$ of $\{u, v\}$ in $K$. Hence $K$ contains a simplex $\sigma^{\prime \prime} \cup\{x\}$ with $x$ being either $u$ or $v$. Under the map $f$, the image of $\sigma^{\prime \prime} \cup\{x\}$ in $K^{\prime}$ is $\left\{u_{0}, \ldots, u_{d}, u\right\}$, hence $\pi(\sigma)$ is well-defined in $K^{\prime}$ in this case too.

Furthermore, in both (i) and (ii) above, $\left(i^{\prime} \circ \pi\right)(\sigma)=\pi(\sigma)$, and $\pi(\sigma)$ is a face of the simplex $\sigma$. Hence $\left(i^{\prime} \circ \pi\right)(\sigma) \cup \mathrm{i} d_{\hat{K}}(\sigma)=\sigma \in \hat{K}$. Putting everything together, we have that $i^{\prime} \circ \pi$ and the identity map i $d_{\hat{K}}$ are contiguous.

\section{B Missing Details from Section 5}

Proof of Proposition 5.1 Let $\mathrm{a}_{z}^{i}$ denote the annotation of a cycle $z$ in $K_{i}$.

Case (i) of elementary inclusion: For $k \neq p$, any $k$-cycle in $K_{i+1}$ was a $k$-cycle in $K_{i}$ and the annotations for $k$-simplices are not altered for $k \neq p$. So, a valid annotation of $K_{i}$ for $k \neq p$ remains so after inclusion of a $p$-simplex $\sigma$. Now consider two $p$-cycles $z$ and $z^{\prime}$ in $K_{i+1}$. We need to show that $\mathrm{a}_{z}^{i+1}=\mathrm{a}_{z^{\prime}}^{i+1}$ if and only if $[z]=\left[z^{\prime}\right]$.

Let $[z]=\left[z^{\prime}\right]$. If $z$ does not include $\sigma$, neither does $z^{\prime}$ and hence both exist in $K_{i}$. In this case

$$
\mathrm{a}_{z}^{i+1}=\left[\mathrm{a}_{z}^{i}, 0\right]=\left[\mathrm{a}_{z^{\prime}}^{i}, 0\right]=\mathrm{a}_{z^{\prime}}^{i+1}
$$

since all $p$-simplices other than $\sigma$ gets the same zero element appended to their annotations while going from $K_{i}$ to $K_{i+1}$. Now consider the case where $z$ includes $\sigma$. Then, $z^{\prime}$ also includes $\sigma$. There is a $p+1$-chain, say $D$, so that $\partial D=z+z^{\prime}$. It follows that $\partial D=(z+\sigma)+\left(z^{\prime}+\sigma\right)$. The $p$-chains $c=z+\sigma$ and $c^{\prime}=z^{\prime}+\sigma$ 
do not include $\sigma$ since it gets canceled under $\mathbb{Z}_{2}$-additions. The $p$-cycle $c+c^{\prime}=\partial D$ is identity in $H_{p}\left(K_{i}\right)$ being a boundary. Therefore, its annotation is zero in $K_{i}$ giving that $\mathrm{a}_{c}=\mathrm{a}_{c^{\prime}}$ in $K_{i}$ and hence in $K_{i+1}$. It follows that $z=c+\sigma$ and $z^{\prime}=c^{\prime}+\sigma$ have identical annotations in $K_{i+1}$.

Now suppose that $[z] \neq\left[z^{\prime}\right]$. If none of $z$ and $z^{\prime}$ include $\sigma$, they exist in $K_{i}$ and by the same logic as above inherit the same annotations from $K_{i}$ which cannot be identical because $K_{i}$ 's annotation is valid. If exactly one of $z$ and $z^{\prime}$ includes $\sigma$, the annotation of one in $K_{i+1}$ will have the last element 1 and that of the other will have 0 . Thus, they will not be identical. Consider the remaining case where both $z$ and $z^{\prime}$ include $\sigma$. Consider the cycle $z+z^{\prime}$ which cannot include $\sigma$ because of $\mathbb{Z}_{2}$-additions. Then, the cycle $z+z^{\prime}$ exists in $K_{i}$ and cannot be in the class [0] because otherwise $\left[z+z^{\prime}\right]$ will remain identity in $H_{p}\left(K_{i+1}\right)$ contradicting $[z] \neq\left[z^{\prime}\right]$ in $K_{i+1}$. Since $\left[z+z^{\prime}\right] \neq[0]$ in $H_{p}\left(K_{i}\right)$, one has $\mathrm{a}_{z}^{i} \neq \mathrm{a}_{z^{\prime}}^{i}$. It follows that

$$
\mathrm{a}_{z}^{i+1}=\left[\mathrm{a}_{z}^{i}, 1\right] \neq\left[\mathrm{a}_{z^{\prime}}^{i}, 1\right]=\mathrm{a}_{z^{\prime}}^{i+1} .
$$

Case (ii) of elementary inclusion: The only annotations altered are for dimensions $p$ and $p-1$. In dimension $p$ the only change is the addition of $\sigma$ along with its zero annotation. In this case, $\sigma$ cannot participate in any $p$-cycle in $K_{i+1}$ because otherwise $\partial \sigma$ should have zero annotation in $K_{i}$. Therefore, annotation for dimension $p$ remains valid in $K_{i+1}$. So, we focus on dimension $p-1$.

Let $z$ and $z^{\prime}$ be two $(p-1)$-cycles with $[z]=\left[z^{\prime}\right]$ in $K_{i+1}$. Observe that both $z$ and $z^{\prime}$ are also $(p-1)$ cycles in $K_{i}$. Recall that a $\partial \sigma$ has been added to all $(p-1)$-simplices with $u$ th element equal to 1 . Hence, the $u$ th element of any $(p-1)$-cycle is exactly equal to the parity of the number of $(p-1)$-simplices in it with $u$ th element equal to 1 . If $[z]=\left[z^{\prime}\right]$ in $K_{i}$, we have $\mathrm{a}_{z}^{i}=\mathrm{a}_{z^{\prime}}^{i}$. In particular, the $u$ th element of $\mathrm{a}_{z}^{i}$ and $\mathrm{a}_{z^{\prime}}^{i}$ are the same implying that $\mathrm{a}_{\partial \sigma}$ has been added with the same parity to $\mathrm{a}_{z}^{i}$ and $\mathrm{a}_{z^{\prime}}^{i}$.

Therefore, $\mathrm{a}_{z}^{i+1}=\mathrm{a}_{z^{\prime}}^{i+1}$. Consider the other case when $[z] \neq\left[z^{\prime}\right]$ in $K_{i}$. Then, there must be a $p$-chain $D$ in $K_{i}$ such that $\partial(D+\sigma)=z+z^{\prime}$ in $K_{i+1}$. We get $\partial D=z+z^{\prime}+\partial \sigma$ and hence $z+z^{\prime}+\partial \sigma=0$ in $K_{i}$. So, the annotation of the cycle $z+z^{\prime}+\partial \sigma$ is zero in $K_{i}$. Since $u$ th element of $a_{\partial \sigma}^{i}$ is 1 , it must be true that $\mathrm{a}_{z}^{i}$ and $\mathrm{a}_{z^{\prime}}^{i}$ differ in the $u$ th element which means

$$
\mathrm{a}_{z}^{i+1}+\mathrm{a}_{z^{\prime}}^{i+1}=\mathrm{a}_{z}^{i}+\mathrm{a}_{z^{\prime}}^{i}+\mathrm{a}_{\partial \sigma}^{i}=0 .
$$

Now suppose that $[z] \neq\left[z^{\prime}\right]$ in $K_{i+1}$. Clearly, $[z] \neq\left[z^{\prime}\right]$ even in $K_{i}$ implying $\mathrm{a}_{z}^{i} \neq \mathrm{a}_{z^{\prime}}^{i}$. If $u$ th elements of $\mathrm{a}_{z}^{i}$ and $\mathrm{a}_{z^{\prime}}^{i}$ are the same, we will have

$$
\mathrm{a}_{z}^{i+1}=\mathrm{a}_{z}^{i}+\mathrm{a}_{\partial \sigma}^{i} \neq \mathrm{a}_{z^{\prime}}^{i}+\mathrm{a}_{\partial \sigma}^{i}=\mathrm{a}_{z^{\prime}}^{i+1}
$$

proving the required. So, assume that $u$ th elements of $\mathrm{a}_{z}^{i}$ and $\mathrm{a}_{z^{\prime}}^{i}$ are different. Without loss of generality, assume that $u$ th element of $\mathrm{a}_{z}^{i}$ is 1 and that of $\mathrm{a}_{z^{\prime}}^{i}$ is 0 . We claim that $\mathrm{a}_{z}^{i}+\mathrm{a}_{\partial \sigma}^{i} \neq \mathrm{a}_{z^{\prime}}^{i}$. Suppose not. Then, by definition of annotation, $[z+\partial \sigma]=\left[z^{\prime}\right]$ in $K_{i}$. Since $[\partial \sigma]=[0]$ in $K_{i+1}$, we have $[z+\partial \sigma]=[z]=\left[z^{\prime}\right]$ in $K_{i+1}$ reaching a contradiction that $[z] \neq\left[z^{\prime}\right]$ in $K_{i+1}$. Therefore, we have $\mathrm{a}_{z}^{i+1} \neq \mathrm{a}_{z^{\prime}}^{i+1}$ because

$$
\mathrm{a}_{z}^{i+1}=\mathrm{a}_{z}^{i}+\mathrm{a}_{\partial \sigma}^{i} \neq \mathrm{a}_{z^{\prime}}^{i}=\mathrm{a}_{z^{\prime}}^{i+1} .
$$

Case for elementary collapse: We already know that $f_{i}$ in this case is a composition of an inclusion $i: K_{i} \hookrightarrow$ $\hat{K}_{i}$ and a collapse $f_{i}^{\prime}: \hat{K}_{i} \rightarrow K_{i+1}$. Since we have argued already that our updates under inclusions

maintain valid annotations, we only show that the collapse under $f_{i}^{\prime}$ also does so.

Recall that $f_{i}^{\prime}$ is implemented with an annotation transfer followed by the actual collapse. Let $\sigma$ be a $p$-simplex where our algorithm adds its annotation to all other $p$-simplices containing a simplex $\tau$ that is a $(p-1)$-face of $\sigma$ adjoining $u$. Adding $\mathrm{a}_{\sigma}$ to all cofaces of $\tau$ of codimension 1 creates a new annotation which is still valid for $K_{i}$ by Proposition 4.4 At the end of all annotation transfers for all $\sigma$, we have a valid annotation for $K_{i}$ with the same cohomology basis such that all vanishing simplices have zero annotation, and each pair of mirror simplices have the same annotation. 
Observe that, under the collapse $\hat{K}_{i} \stackrel{f_{i}^{\prime}}{\rightarrow} K_{i+1}$, the set of vanishing simplices are exactly those simplices $\sigma$ for which $f_{i}^{\prime}(\sigma)$ has a lower dimension than $\sigma$. A pair of mirror simplices $\tau$ and $\tau^{\prime}$ are those that satisfy that $f_{i}^{\prime}(\tau)=f_{i}^{\prime}\left(\tau^{\prime}\right)$ (i.e, the simplex $\tau^{\prime}$ containing $v$ coincides with its mirror partner $\tau$ containing $u$ ). Hence after the collapse, if $f_{i}^{\prime}(\sigma)$ is a $p$-simplex for any $p$-simplex $\sigma \in \hat{K}_{i}$, we have $\mathrm{a}_{\sigma}=\mathrm{a}_{f_{i}^{\prime}(\sigma)}$ by construction. We can now finish the argument that this induced annotation for $K_{i+1}$ is valid.

Let $z$ and $z^{\prime}$ be any two $p$-cycles in $K_{i+1}$. Let $w$ and $w^{\prime}$ be two $p$-cycles in $\hat{K}_{i}$ so that $f_{i}^{\prime}(w)=z$ and $f_{i}^{\prime}\left(w^{\prime}\right)=z^{\prime}$. Then, $[w]=\left[w^{\prime}\right]$ if and only if $[z]=\left[z^{\prime}\right]$ since $f_{i_{*}}^{\prime}: H_{p}\left(K_{i}\right) \rightarrow H_{p}\left(K_{i+1}\right)$ is an isomorphism (Proposition 4.2). With the modified annotation of $\hat{K}_{i}$ we have $\mathrm{a}_{w}^{i}=\mathrm{a}_{w^{\prime}}^{i}$ if and only if $[w]=\left[w^{\prime}\right]$. Therefore, $[z]=\left[z^{\prime}\right]$ in $K_{i+1}$ if and only if $\mathrm{a}_{w}^{i}=\mathrm{a}_{w^{\prime}}^{i}$. The only simplices where $z$ and $w$ differ are either vanishing simplices or mirror simplices. In the first case, the annotation is zero and in the second case the annotations are the same. So, $\mathrm{a}_{w}^{i}=\mathrm{a}_{z}^{i+1}$. Similarly, $\mathrm{a}_{w^{\prime}}^{i}=\mathrm{a}_{z^{\prime}}^{i+1}$. Therefore, $\mathrm{a}_{z}=\mathrm{a}_{z^{\prime}}$ if and only if $[z]=\left[z^{\prime}\right]$ in $K_{i+1}$. This proves that the annotation for $K_{i+1}$ is valid.

Proof of Case (ii) of Proposition 5.2 In this case, a $(p-1)$-cycle is killed as we add $\sigma$, so in the reverse direction a cocycle is created. As before, assume that $\left[z_{1}\right], \ldots,\left[z_{g}\right]$ be a homology basis for $H_{p-1}(K)$. By assumption, the $u$ th element in the annotation has been zeroed out. Let $\phi$ be the cocycle given by $f$ and $\phi_{i}^{\prime}$ where $i \in\{1, \ldots, u-1\}$. Then, as before we get

$$
\begin{aligned}
{\left[\phi\left(z_{1}\right), \ldots, \phi\left(z_{g}\right)\right] } & =\left[\phi_{i}^{\prime}\left(f_{\#}\left(z_{1}\right), \ldots, \phi_{i}^{\prime}\left(f_{\#}\left(z_{g}\right)\right)\right]\right. \\
& =\left[\phi_{i}^{\prime}\left(z_{1}\right), \ldots, \phi_{i}^{\prime}\left(z_{g}\right)\right]
\end{aligned}
$$

Consider any entry $\phi_{i}^{\prime}\left(z_{j}\right)$ in the last vector. If $\phi_{i}\left(z_{j}\right)$ has $u$ th element 0 , then we must have $\phi_{i}^{\prime}\left(z_{j}\right)=\phi_{i}\left(z_{j}\right)$. This is because, in that case, $z_{j}$ has even number of simplices whose annotations have $u$ th element 1 . Then, according to the update algorithm the annotation $\mathrm{a}_{\partial \sigma}$ is added to the simplices in $z_{j}$ only even number of times in total maintaining $\phi_{i}^{\prime}\left(z_{j}\right)=\phi_{i}\left(z_{j}\right)$.

If $\phi_{i}\left(z_{j}\right)$ has $u$ th element 1 , we consider the cycle $z_{j}+\partial \sigma$ and observe that $\left[z_{j}+\partial \sigma\right]=\left[z_{j}\right]$ in $H_{p-1}\left(K^{\prime}\right)$. Then, $\phi_{i}^{\prime}\left(z_{j}\right)=\phi_{i}^{\prime}\left(z_{j}+\partial \sigma\right)$ since $\phi_{i}^{\prime}$ is derived from a valid annotation for $K^{\prime}$. The cycle $z_{j}$ has odd number of simplices whose annotations have $u$ th element 1 as $\phi_{i}\left(z_{j}\right)$ has $u$ th element 1 . So, a $\partial \sigma$ has been added odd number of times to $\mathrm{a}_{z_{j}}$ and hence even number of times to $\mathrm{a}_{z_{j}+\partial \sigma}$. This implies that $\phi_{i}^{\prime}\left(z_{j}+\partial \sigma\right)=\phi_{i}\left(z_{j}\right)$ which leads to $\phi_{i}^{\prime}\left(z_{j}\right)=\phi_{i}\left(z_{j}\right)$. This immediately gives $\left[\phi\left(z_{1}\right), \ldots, \phi\left(z_{g}\right)\right]=\left[\phi_{i}^{\prime}\left(z_{1}\right), \ldots, \phi_{i}^{\prime}\left(z_{g}\right)\right]$ which we are required to prove.

\section{The Size of $\mathcal{R}^{\alpha(1+\varepsilon)^{k}}\left(V_{k}\right)$}

We argue that we can construct every $V_{k}$ in such way that each $\mathcal{R}^{\alpha(1+\varepsilon)^{k}}\left(V_{k}\right)$ is of size linear in $n$. We compute $V_{k+1}$ such that it is a $\delta$-net of $V_{k}$ for $\delta=\frac{\alpha \varepsilon^{2}}{2}(1+\varepsilon)^{k-1}$ by the following standard greedy approach: Let $D(\cdot, \cdot)$ denote the metric on the set of input points $P$ (and thus $V_{k} \mathrm{~s}$ ). Starting with $V_{k+1}=\emptyset$, pick an arbitrary vertex from $V_{k}$ and add it to $V_{k+1}$. In the $i$ th round, there are already $i$ points in $V_{k+1}$. We identify the point $u$ from $V_{k}$ whose minimum distance to points in $V_{k+1}$ is the largest. We stop when either $D\left(u, V_{k+1}\right) \leq \delta$ or $V_{k+1}=V_{k}$. By construction, when this process terminates, any point in $V_{k}$ is within $\delta$ distance to some point in $V_{k+1}$, and no two points in $V_{k+1}$ are within $\delta$ distance. A naïve implementation of the above procedure takes $O\left(n^{2}\right)$ time. One can also compute the $\delta$-net $V_{k+1}$ more efficiently in $O(n \log n)$ time (see, e.g, [21]). However, we remark that this step does not form a bottleneck in the time complexity as computing persistence diagrams takes time cubic in the number of simplices. 
Proposition C.1 Suppose the set of input points $V$ are from a metric space with doubling dimension d. For $V_{k}$ s constructed as described above, the number of p-simplices in $\mathcal{R}^{\alpha(1+\varepsilon)^{k+1}}\left(V_{k+1}\right)$ is $O\left(\left(\frac{1}{\varepsilon}\right)^{O(d p)} \cdot n\right)$ for $0 \leq \varepsilon \leq 1$.

Proof: For simplicity, set $r:=\alpha(1+\varepsilon)^{k+1}$; note that $\delta=\frac{\varepsilon^{2}}{2(1+\varepsilon)^{2}} r$. We first prove that there are $O\left(\left(\frac{1}{\varepsilon}\right)^{O(d)}\right)$ number of edges for each vertex in $V_{k+1}$. Specifically, consider a node $u \in V_{k+1}$ : it will be connected to all other vertices in $V_{k+1}$ that are within distance $r$ to $u$. Since $V_{k+1}$ is a $\delta$-net of $V_{k}$, every node in $V_{k+1}$ has a ball centered at it with radius $\delta / 2$ that is empty of other points in $V_{k+1}$. Since the points are from a metric space with doubling dimension $d$, we can pack only $O\left(\left(\frac{r}{\delta / 2}\right)^{d}\right)=O\left(\left(\frac{4(1+\varepsilon)^{2}}{\varepsilon^{2}}\right)^{d}\right)=O\left(\left(\frac{4}{\varepsilon^{2}}\right)^{d}\right)$ (for $0 \leq \varepsilon \leq 1)$ number of balls of radius $\delta / 2$ in a ball of radius $r$. This means that there are only $O\left(\left(\frac{1}{\varepsilon}\right)^{2 d}\right)$ number of edges containing $u$, where the big- $O$ notation hides terms exponential in $d$. It then follows that the number of $p$-simplices containing $u$ is $O\left(\left(\frac{1}{\varepsilon}\right)^{2 d p}\right)$. Since there are $\left|V_{k+1}\right| \leq n$ number of vertices in $V_{k+1}$, the total number of $p$-simplices is bounded by $O\left(\left(\frac{1}{\varepsilon}\right)^{O(d p)} n\right)$ as claimed. 